\title{
Multiple Attribute Strategic Weight Manipulation With Minimum Cost in a Group Decision Making Context With Interval Attribute Weights Information
}

\author{
Yating Liu, Yucheng Dong ${ }^{(\mathbb{C}}$, Haiming Liang, Francisco Chiclana ${ }^{(\mathbb{D}}$, and Enrique Herrera-Viedma ${ }^{(\mathbb{D}}$
}

\begin{abstract}
In multiple attribute decision making (MADM), strategic weight manipulation is understood as a deliberate manipulation of attribute weight setting to achieve a desired ranking of alternatives. In this paper, we study the strategic weight manipulation in a group decision making (GDM) context with interval attribute weight information. In GDM, the revision of the decision makers' original attribute weight information implies a cost. Driven by a desire to minimize the cost, we propose the minimum cost strategic weight manipulation model, which is achieved via optimization approach, with the mixed 0-1 linear programming model being proved appropriate in this context. Meanwhile, some desired properties to manipulate a strategic attribute weight based on the ranking range under interval attribute weight information are proposed. Finally, numerical analysis and simulation experiments are provided with a twofold aim: 1) to verify the validity of the proposed models and 2) to show the effects of interval attribute weights information and the unit cost, respectively, on the cost to manipulate strategic weights in the MADM in a group decision context.
\end{abstract}

Index Terms-Interval attribute weight information, minimum cost, multiple attribute decision making (MADM), strategic weight manipulation.

\section{NOMENCLATURE}

The main notations in this paper are as follows.

$\begin{array}{ll}X & \text { Set of alternatives. } \\ A & \text { Set of attributes. } \\ E & \text { Set of experts. } \\ V=\left[v_{i j}\right]_{n \times m} & \text { Decision matrix. }\end{array}$

Manuscript received June 16, 2018; revised July 23, 2018; accepted October 2, 2018. Date of publication October 26, 2018; date of current version September 16, 2019. This work was supported in part by National Science Foundation of China under Grant 71571124, Grant 71871149, and Grant 71601133; in part by Sichuan University under Grant sksyl201705 and Grant 2018hhs-58; and in part by FEDER Funds under Grant TIN201675850-R. This paper was recommended by Associate Editor C. Zhang. (Corresponding author: Yucheng Dong.)

Y. Liu, Y. Dong, and H. Liang are with the Business School, Sichuan University, Chengdu 610065, China (e-mail: yatingliu @ stu.scu.edu.cn; ycdong@scu.edu.cn; hmliang@scu.edu.cn).

F. Chiclana is with the Centre for Computational Intelligence, Faculty of Technology, De Montfort University, Leicester LE1 9BH, U.K. (e-mail: chiclana@dmu.ac.uk).

E. Herrera-Viedma is with the Department of Computer Science and Artificial Intelligence, University of Granada, 18071 Granada, Spain, and also with the Department of Electrical and Computer Engineering, Faculty of Engineering, King Abdulaziz University, Jeddah 21589, Saudi Arabia (e-mail: viedma@decsai.ugr.es).

Color versions of one or more of the figures in this paper are available online at http://ieeexplore.ieee.org.

Digital Object Identifier 10.1109/TSMC.2018.2874942

$\bar{V}=\left[\bar{v}_{i j}\right]_{n \times m}$
$D_{w}\left(x_{i}\right)$
$\mathbf{w}$
$\mathbf{w}^{\mathbf{k}}$
$\overline{\mathbf{w}^{k}}$
$r_{w}\left(x_{h}\right)$
$W$
$\underline{r}_{w \in W}\left(x_{h}\right)$
$\bar{r}_{\boldsymbol{w} \in W}\left(x_{h}\right)$

$R_{w \in W}\left(x_{h}\right)=\left[\underline{r}_{w \in W}\left(x_{h}\right)\right.$, $\left.\bar{r}_{w \in W}\left(x_{h}\right)\right]$ $R_{w \in W}^{\mathrm{WA}}\left(x_{h}\right)=\left[\underline{r}_{w \in W}^{\mathrm{WA}}\left(x_{h}\right)\right.$, Ranking range under the set of $\left.\bar{r}_{w \in W}^{\mathrm{WA}}\left(x_{h}\right)\right]$

$R_{w \in W}^{\mathrm{OWA}}\left(x_{h}\right)=\left[\underline{r}_{\boldsymbol{w} \in W}^{\mathrm{OWA}}\left(x_{h}\right)\right.$, Ranking range under the set of $\left.\bar{r}_{w \in W}^{\mathrm{OWA}}\left(x_{h}\right)\right]$

$S$

$\underline{r}_{w \in S}\left(x_{h}\right)$

$\bar{r}_{w \in S}\left(x_{h}\right)$

$R_{w \in S}^{\mathrm{WA}}\left(x_{h}\right)=\left[\underline{r}_{w \in S}^{\mathrm{WA}}\left(x_{h}\right)\right.$,

$\left.\bar{r}_{w \in S}^{\mathrm{WA}}\left(x_{h}\right)\right]$

$R_{w \in S}^{\mathrm{OWA}}\left(x_{h}\right)\left[\underline{r}_{w \in S}^{\mathrm{OWA}}\left(x_{h}\right)\right.$,

$\left.\bar{r}_{\boldsymbol{w} \in S}^{\mathrm{OWA}}\left(x_{h}\right)\right]$
Standardized decision matrix.

Evaluation function of alternative $x_{i}$ with weight vector $\mathbf{w}$.

Weight vector of attribute weights. Original attribute weight vector over expert $e_{k}$.

Revised attribute weight vector associated with expert $e_{k}$.

Ranking of alternative $x_{h}$ under attribute weight vector $\mathbf{w}$.

Attribute weights set without any constraint.

Best ranking of alternative $x_{h}$ under the set of attribute weights $W$.

Worst ranking of alternative $x_{h}$ under the set of attribute weights $W$.

Ranking range under the set of attribute weights $W$ associated with the WA operator attribute weights $W$ associated with the OWA operator.

Set of interval information of attribute weights.

Best ranking of alternative $x_{h}$ under the set of interval attribute weights $S$.

Worst ranking of alternative $x_{h}$ under the set of interval attribute weights $S$.

Ranking range under the set of interval attribute weights $S$ associated with the WA operator.

Ranking range under the set of interval attribute weights $S$ associated with the OWA operator.

\section{INTRODUCTION}

$\mathbf{M}$ ULTIPLE attribute decision making (MADM) aims to obtain a ranking of alternatives based on their 
evaluation information regarding multiple attributes. MADM has received increasing attention in decision analysis [22], [37], [45], [46], and it has been applied in a wide range of fields [5], [8], [19], [33].

Attribute weights play an important role in the resolution of MADM problems [28], [29]. Until now, there exist many approaches in the specialized literature on how to obtain the attribute weights in MADM. The existing approaches can be mainly divided into three categories [12].

1) The subjective approach obtains the attribute weights according to the decision makers' subjective preference information on the set of attributes. For example, Doyle et al. [15] proposed a direct rating method and a point allocation method; Barron and Barrett [1] investigated three rank-ordered methods, while Roberts and Goodwin [32] provided a rank order distribution approach.

2) The objective approach determines the attribute weights by using objective decision matrix information and the entropy method [47]; or a TOPSIS-based method [48]; or some other mathematical programmingbased method [7], [35].

3) The integrated approach obtains the attribute weights according to both the decision makers' subjective preference information and the objective decision matrix information. For example, Cook and Kress [10] proposed a preference-aggregation model, while Fan et al. [16], Horsky and Rao [20], and Pekelman and Sen [30] constructed optimization-based models.

Strategic manipulation or noncooperative behavior in decision making describes those situations in which some decision makers dishonestly express opinions to enhance the chances of obtaining their most preferred alternatives. Strategic manipulation is a common phenomenon and has been analyzed in depth in different decision contexts. For example, Pelta and Yager [31] and Yager [42], [43] have proposed aggregation approaches to defend against the strategic manipulation in group decision making (GDM); where as Dong et al. [13], Palomares et al. [26], and $\mathrm{Xu}$ et al. [41] have investigated how to detect and manage a series of noncooperative behaviors in GDM consensus reaching processes from different perspectives.

As mentioned above, approaches to set attribute weights have been investigated intensively, however, in these approaches decision makers are assumed to be honest when expressing their preferences regarding attribute weights. Recently, Dong et al. [12] proposed the concept of strategic weight manipulation, in which a decision maker can be dishonest in the sense of setting attribute weights strategically to obtain his/her desired ranking of alternatives.

Although this paper by Dong et al. is useful in MADM, there still exist issues that need to be addressed.

1) In [12], the strategic weight manipulation was investigated in an individual decision making context. However, the increasing complexity of decision environments means that many practical decisions involve multiple decision makers. Additionally, the strategic weight manipulation investigated in [12] assumed no constraints on the weights and consequently the strategic attribute weights could be set freely as anyone of the domain values. However, decision makers often will present some attribute weight information [6], [21], [23], [27], and thus, some attribute weight information is partially known or subject to certain constraints. Therefore, it is necessary to investigate the strategic weight manipulation in a group decision context in which attribute weight information is partially known.

2) When decision makers provide partially attribute weights information in a group decision context, it is more challenging for a manipulator to strategically set attribute weights because some decision makers may be reluctant to change their original attribute weights preferences. As a result, the manipulator needs to take some cost for decision makers to revise their original attribute weight preferences. Driven by a desire to minimize the cost, it is necessary to investigate the strategic weight manipulation with minimum cost.

In order to address these two issues, this paper proposes the strategic weight manipulation with minimum cost in a GDM context with interval attribute weight information. The proposed methodology to achieve this consists of the following main steps.

1) Attribute weights are considered partially known, and they are described by numerical intervals, i.e., interval attribute weights information is assumed. Additionally, multiple decision makers are assumed to be involved in the strategic weight manipulation. Following these assumption, this paper develops a new strategic weight manipulation model in a group decision context with interval attribute weights information.

2) A minimum cost model is developed to strategically set the attribute weights, by revising the decision makers' original preferences of attribute weights to obtain a desired ranking of alternatives. Meanwhile, some desired properties with zero cost for manipulating strategic attribute weights are explored. Simulation experiments with real data are provided to show the effects of the interval attribute weight information and the unit cost, respectively, in the cost to manipulate strategic weights in the MADM in a group context.

The remainder of this paper is organized as follows. Section II introduces some basic concepts regarding the MADM. Mixed 0-1 linear programming models to set a multiple attribute strategic weight vector with minimum cost are constructed in Section III. Section IV presents numerical analysis and simulation experiments to justify the proposal put forward in this paper. Concluding remarks and future research agenda are included in Section V.

\section{PRELIMINARIES}

This section introduces some basic knowledge regarding MADM and attribute weights. 


\section{A. Classical MADM Problem}

A classical MADM problem can be described as follows: Let $X=\left\{x_{1}, \ldots, x_{n}\right\}$ be a finite set of alternatives, $A=\left\{a_{1}, \ldots, a_{m}\right\}$ a set of predefined attributes, and $\mathbf{w}=$ $\left(w_{1}, w_{2}, \ldots, w_{m}\right)^{T}$ the weight vector of the attributes, where $w_{j} \geq 0$ and $\sum_{j=1}^{m} w_{j}=1$. Let $V=\left[v_{i j}\right]_{n \times m}$ be the decision matrix, where $v_{i j}$ denotes the attribute value associated with alternative $x_{i} \in X$ and attribute $a_{j} \in A$. The resolution process of an MADM problem includes, generally, two steps.

1) Normalization Phase: Attributes are split into two categories: 1) benefit attributes and 2) cost attributes. The decision matrix $V=\left[v_{i j}\right]_{n \times m}$ is transformed into a normalized decision matrix $\bar{V}=\left[\bar{v}_{i j}\right]_{n \times m}$, where

$$
\bar{v}_{i j}=\frac{v_{i j}-\min _{i}\left(v_{i j}\right)}{\max _{i}\left(v_{i j}\right)-\min _{i}\left(v_{i j}\right)}
$$

if $a_{j} \in A$ is a benefit attribute, while

$$
\bar{v}_{i j}=\frac{\max _{i}\left(v_{i j}\right)-v_{i j}}{\max _{i}\left(v_{i j}\right)-\min _{i}\left(v_{i j}\right)}
$$

if $a_{j} \in A$ is a cost attribute.

2) Ranking of Alternatives: Alternatives are ranked by associating them with an evaluation value $D_{w}\left(x_{i}\right)$, which is computed by a decision function $F$ that assigns an overall evaluation to each alternative, i.e., $D_{w}\left(x_{i}\right)=F_{w}\left(\bar{v}_{i 1}, \bar{v}_{i 2}, \ldots, \bar{v}_{i m}\right)$, with $\mathbf{w}=\left(w_{1}, w_{2}, \ldots, w_{m}\right)^{T}$ being the attribute weight vector. It is worth mentioning out at this point that the alternatives' overall evaluation is frequently derived by fusing their attributes normalized decision values, i.e., by using as function $F$ an aggregation operator such as the weighted average (WA) or the ordered weighted average (OWA) operators [38], [44], which would result, respectively, in

$$
D_{w}\left(x_{i}\right)=\mathrm{WA}_{\boldsymbol{w}}\left(\bar{v}_{i 1}, \bar{v}_{i 2}, \ldots, \bar{v}_{i m}\right)=\sum_{j=1}^{m} w_{j} \bar{v}_{i j}
$$

where $w_{j}$ is the weight associated with the attribute $a_{j}$

$$
D_{w}\left(x_{i}\right)=\mathrm{OWA}_{\boldsymbol{w}}\left(\bar{v}_{i 1}, \bar{v}_{i 2}, \ldots, \bar{v}_{i m}\right)=\sum_{j=1}^{m} w_{j} \bar{v}_{i(j)}
$$

where $\bar{v}_{i(j)}$ is the $j$ th largest value in $\left\{\bar{v}_{i 1}, \bar{v}_{i 2}, \ldots, \bar{v}_{i m}\right\}$, and $w_{j}$ is the weight associated with the $j$ th largest value in $\left\{\bar{v}_{i 1}, \bar{v}_{i 2}, \ldots, \bar{v}_{i m}\right\}$.

There exist various approaches to rank the alternatives. However, as this paper is a continuation of the study presented in [12], the ranking approach used there is also employed here: let $Q_{h, w}=\left\{x_{i} \mid D_{w}\left(x_{i}\right)>D_{w}\left(x_{h}\right), i=1,2, \ldots, n\right\}$ be the set of the alternatives whose decision evaluation value is greater than that of the alternative $x_{h}$, and $\left|Q_{h, w}\right|$ its cardinality. Then, the ranking position of the alternative $x_{h}$ is

$$
r_{w}\left(x_{h}\right)=\left|Q_{h, w}\right|+1 \text {. }
$$

\section{B. Research Problem: Attribute Weights in Group Decision Context With Interval Attribute Weight Information}

As mentioned in Dong et al. [12], the setting of attribute weights has an important effect on the ranking of alternatives. Thus, a manipulator may strategically set the attribute weights to attain his/her desired ranking in the MADM.

Generally, in real-life MADM problems, the decision matrix $V=\left[v_{i j}\right]_{n \times m}$ is considered as providing representing objective information, with the attribute weights being set by one or more decision makers.

We make the following assumption.

1) Let $E=\left\{e_{1}, e_{2}, \ldots, e_{l}\right\}$ be a set of decision makers and let $\mathbf{w}^{\mathbf{k}}=\left(w_{1}^{k}, w_{2}^{k}, \ldots, w_{m}^{k}\right)^{T}$ be the weight vector of the attributes associated with the decision maker $e_{k} \in E$, where $w_{j}^{k} \geq 0$ and $\sum_{j=1}^{m} w_{j}^{k}=1$. The attribute weight vector $\mathbf{w}=\left(w_{1}, w_{2}, \ldots, w_{m}\right)^{T}$ is determined as the average of all decision makers' corresponding attribute weight vectors

$$
w_{j}=\frac{\sum_{k=1}^{l} w_{j}^{k}}{l} .
$$

In MADM problems, because of time pressure or limited expertise, some decision makers might not be able to provide precise attribute weights but incomplete attribute weights instead [6], [21], [23], [27], i.e., some information on attributes weights may be unknown or represented as interval values. Usually, the basic forms of incomplete attribute weights include weak ranking, strict ranking, ranking multiples, interval form, ranking differences, and bounded (see [21], [23], and [27]). In this paper, we consider interval attribute weights, i.e., the attribute weights are in some numerical intervals. Then, we make the following assumption.

2) The attribute weight vector $\mathbf{w}^{\mathbf{k}}=\left(w_{1}^{k}, w_{2}^{k}, \ldots, w_{m}^{k}\right)^{T}$, associated with the decision maker $e_{k} \in E$, is an interval weight vector, i.e.,

$$
w_{j}^{k}=\left[\begin{array}{ll}
I_{j}^{k,-}, & I_{j}^{k,+}
\end{array}\right]
$$

where $0 \leq I_{j}^{k,-} \leq I_{j}^{k,+} \leq 1$. When conditions $\sum_{i=1}^{m} I_{i}^{k,+}-\max _{j}\left(I_{j}^{k,+}-I_{j}^{k,-}\right) \geq 0$ and $\sum_{i=1}^{m} I_{i}^{k,-}+$ $\max _{j}\left(I_{j}^{k,+}-I_{j}^{k,-}\right) \leq 1$ are verified, $\mathbf{w}^{\mathbf{k}}$ is said to be a normalized interval weight vector [37]. These conditions guarantee that there exists a weight vector $\mathbf{w}=\left(w_{1}, w_{2}, \ldots, w_{m}\right)^{T}$ such that $\sum_{j=1}^{m} w_{j}=1$ and $I_{j}^{k,-} \leq w_{j} \leq I_{j}^{k,+}(\forall j)$.

When the decision makers have interval information of attribute weights, setting strategic attribute weights carries a cost as the original attribute weight information has to be revised, i.e., modified. Inspired by the classical minimum cost model [2], [3], in this paper, we study the multiple attribute strategic weight manipulation with minimum cost in a GDM context with interval attribute weights information. Minimum cost strategic weight manipulation (MCSWM) will be formulated and discussed in the next section. 


\section{Multiple Attribute Strategic Weight Manipulation With Minimum Cost}

This section contains 1) the strategic weight manipulation with minimum cost in MADM, 2) an approach based on the mixed 0-1 linear programming to obtain its optimal solution, and 3) some desired properties.

\section{A. Basic Ideas and Model}

In this section, we introduce some basic ideas and construct an optimization-based model with minimum cost to find out the manipulator's strategic weight vector to obtain his/her desired ranking of alternative(s).

In this paper, without loss of generality, we assume that the manipulator wants to manipulate the alternatives $\left\{X_{h \in G} \mid G \subseteq\right.$ $\{1,2, \ldots, n\}\}$, to which the attribute weight vector $\mathbf{w}=$ $\left(w_{1}, w_{2}, \ldots, w_{m}\right)^{T}$ is to be strategically set.

Let $\mathbf{w}^{\mathbf{k}}=\left(w_{1}^{k}, w_{2}^{k}, \ldots, w_{m}^{k}\right)^{T}$ be the original normalized interval attribute weights vector associated with the decision maker $e_{k}$. In order to strategically set the attribute weight vector $\mathbf{w}=\left(w_{1}, w_{2}, \ldots, w_{m}\right)^{T}$, the manipulator hopes that the decision makers can revise their original interval information regarding attribute weight vectors. Let us denote $\overline{\mathbf{w}^{\mathbf{k}}}=$ $\left(\overline{w_{1}^{k}}, \overline{w_{2}^{k}}, \ldots, \overline{w_{m}^{k}}\right)^{T}$ as the revised attribute weight vector associated with the decision maker $e_{k}$, where $\sum_{j=1}^{m} \overline{w_{j}^{k}}=1$ and $0 \leq \overline{w_{j}^{k}} \leq 1$. The difference between the original and the revised attribute weight vector associated with the decision maker $e_{k}$ can be measured by

$$
d\left(\boldsymbol{w}^{k}, \overline{\boldsymbol{w}^{k}}\right)=\sum_{j=1}^{m} d\left(w_{j}^{k}, \overline{w_{j}^{k}}\right)
$$

where

$$
d\left(w_{j}^{k}, \overline{w_{j}^{k}}\right)= \begin{cases}I_{j}^{k,-}-\overline{w_{j}^{k}}, & 0 \leq \overline{w_{j}^{k}}<I_{j}^{k,-} \\ 0, & I_{j}^{k,-} \leq \overline{w_{j}^{k}} \leq I_{j}^{k,+} \\ \overline{w_{j}^{k}}-I_{j}^{k,+}, & I_{j}^{k,+}<\overline{w_{j}^{k}} \leq 1 .\end{cases}
$$

Motivated by the minimum cost model, setting strategic attribute weights means the manipulator needs to take some cost for the decision makers to revise their original interval attribute weights information. Let $f_{k}$ be the unit cost to revise the decision maker $e_{k}$ 's attribute weight. The unit cost is a basic concept of minimum cost GDM models [17], [18], [24], [51], [53] and refers to the cost for the decision makers adjusting the unit opinions. Usually, the unit cost can be measured by money, time, and so on. In practical GDM context, the manipulator often assumes the cost to persuade the decision makers in changing their opinions, and the finalized measurement for the cost is determined by the specified decision making problem. Generally, the greater the distance of experts changing their opinions, the greater the cost. Thus, the cost function of revising the decision maker $e_{k}$ 's attribute weight can be defined as the product of the unit cost and distance of opinions changing: $f_{k} d\left(\boldsymbol{w}^{k}, \overline{w^{k}}\right)$.
Thus, the cost function of revising all the decision makers' attribute weights can be denoted as

$$
\sum_{k=1}^{l} f_{k} d\left(\boldsymbol{w}^{k}, \overline{\boldsymbol{w}^{\boldsymbol{k}}}\right)=\sum_{k=1}^{l} \sum_{j=1}^{m} f_{k} d\left(w_{j}^{k}, \overline{w_{j}^{k}}\right) .
$$

It is assumed that the manipulator aims to minimize the cost, that is

$$
\min \sum_{k=1}^{l} \sum_{j=1}^{m} f_{k} d\left(w_{j}^{k}, \overline{w_{j}^{k}}\right) .
$$

Meanwhile, following (6), the attribute weight vector strategically set by the manipulator is determined as follows:

$$
w_{j}=\frac{\sum_{k=1}^{l} \overline{w_{j}^{k}}}{l} .
$$

Moreover, we assume the set of the alternatives that the manipulator wants to manipulate is $\left\{x_{h \in G} \mid G \subseteq\{1,2, \ldots, n\}\right\}$, and the manipulator's desired ranking of the alternatives in $\left\{x_{h \in G} \mid G \subseteq\{1,2, \ldots, n\}\right\}$ is $\left\{r^{*}\left(x_{h \in G}\right) \mid G \subseteq\right.$ $\{1,2, \ldots, n\}\}$, i.e.,

$$
r_{w}\left(x_{h}\right)=r^{*}\left(x_{h}\right)
$$

where $h \in G$. Let $|G|$ be the number of the alternatives in the set $\left\{x_{h \in G} \mid G \subseteq\{1,2, \ldots, n\}\right\}$, and it is assumed that $|G| \geq 1$ in this paper.

Based on (8)-(13), we construct the MCSWM model to set the strategic weight vector as follows:

$$
\left\{\begin{array}{l}
\min \sum_{k=1}^{l} \sum_{j=1}^{m} f_{k} d\left(w_{j}^{k}, \quad \overline{w_{j}^{k}}\right) \\
\text { s.t. }\left\{\begin{array}{l}
r_{w}\left(x_{h}\right)=r^{*}\left(x_{h}\right), \quad(h \in G) \\
\mathbf{w}=\left(w_{1}, w_{2}, \ldots, w_{m}\right)^{T} \\
w_{j}=\frac{\sum_{k=1}^{l} \overline{w_{j}^{k}}}{l}, \quad(j=1,2, \ldots, m) \\
\sum_{j=1}^{m} \overline{w_{j}^{k}}=1, \quad(k=1,2, \ldots, l) \\
0 \leq \overline{w_{j}^{k}} \leq 1, \quad(j=1,2, \ldots, m) \\
w_{j}^{k}=\left[I_{j}^{k,-}, I_{j}^{k,+}\right], \quad(k=1,2, \ldots, l ; j=1,2, \ldots, m)
\end{array}\right.
\end{array}\right.
$$

where $\overline{w_{j}^{k}},(k=1,2, \ldots, l ; j=1,2, \ldots, m)$ are the decision variables.

\section{B. Solving the Minimum Cost Strategic Weight Manipulation Model via Mixed 0-1 Linear Programming}

In this section, we continue to use a mixed 0-1 linear programming methodology to obtain the optimal solution to the MCSWM [model (14)].

In order to transform model (14) into a mixed 0-1 linear programming, binary variable $y_{i h} \in\{0,1\}$ and a large enough number $M$ are introduced. Then, we have the following results.

1) $x_{i} \succ x_{h}$ if and only if $y_{i h}=1$ under the conditions: $D_{w}\left(x_{i}\right)>D_{w}\left(x_{h}\right)-\left(1-y_{i h}\right) M$ and $D_{w}\left(x_{i}\right) \leq D_{w}\left(x_{h}\right)+$ $y_{i} M$. 
2) $x_{i \sim} \prec x_{h}$ if and only if $y_{i h}=0$ under the following conditions: $D_{w}\left(x_{i}\right) \leq D_{w}\left(x_{h}\right)+y_{i h} M$ and $D_{w}\left(x_{i}\right)>$ $D_{w}\left(x_{h}\right)-\left(1-y_{i h}\right) M$.

The following lemmas are proposed.

Lemma 1: For decision function with $F$ the WA operator as per (3), if there exists $\mathbf{w}^{*}=\left(w_{1}^{*}, w_{2}^{*}, \ldots, w_{m}^{*}\right)^{T}$ satisfying constraint conditions

$$
\begin{aligned}
& \int \sum_{j=1}^{m} w_{j}^{*} \bar{v}_{i j}>\sum_{j=1}^{m} w_{j}^{*} \bar{v}_{h j}-\left(1-y_{i h}\right) M \\
& (i=1,2, \ldots, n ; h \in G) \\
& \sum_{j=1}^{m} w_{j}^{*} \bar{v}_{i j} \leq \sum_{j=1}^{m} w_{j}^{*} \bar{v}_{h j}+y_{i h} M \\
& (i=1,2, \ldots, n ; h \in G) \\
& y_{i h}=1 \text { or } 0, \quad(i=1,2, \ldots, n ; h \in G) \\
& \sum_{i=1}^{n} y_{i h}+1=r^{*}\left(x_{h}\right), \quad(h \in G) \\
& w_{j}^{*}=\frac{\sum_{k=1}^{l} \overline{w_{j}^{*, k}}}{l}, \quad(j=1,2, \ldots, m) \\
& \sum_{j=1}^{m} \overline{w_{j}^{*, k}}=1, \quad(k=1,2, \ldots, l) \\
& 0 \leq \overline{w_{j}^{*, k}} \leq 1, \quad(j=1,2, \ldots, m) \\
& w_{j}^{k}=\left[I_{j}^{k,-}, I_{j}^{k,+}\right], \quad(k=1,2, \ldots, l ; j=1,2, \ldots, m) \\
& d\left(\overline{w_{j}^{*, k}}, w_{j}^{k}\right)=\left\{\begin{array}{l}
I_{j}^{k,-}-\overline{w_{j}^{*, k}}, \quad 0 \leq \overline{w_{j}^{*, k}} \leq I_{j}^{k,-} \\
0, I_{j}^{k,-}<\overline{w_{j}^{*, k}} \leq I_{j}^{k,+} \\
\quad(k=1,2, \ldots, l ; j=1,2, \ldots, m) \\
\overline{w_{j}^{*, k}}-I_{j}^{k,+}, \quad I_{j}^{k,+}<\overline{w_{j}^{*, k}} \leq 1
\end{array}\right.
\end{aligned}
$$

then, $r_{w^{*}}\left(x_{h}\right)=r^{*}\left(x_{h}\right),(h \in G)$.

The proof of Lemma 1 is provided in Appendix A.

Lemma 2: For decision function with $F$ the OWA operator as per (4), if there exists $\mathbf{w}^{*}=\left(w_{1}^{*}, w_{2}^{*}, \ldots, w_{m}^{*}\right)^{T}$ satisfying constraint conditions (17)-(25)

$$
\left\{\begin{array}{c}
\sum_{j=1}^{m} w_{j}^{*} \bar{v}_{i(j)}>\sum_{j=1}^{m} w_{j}^{*} \bar{v}_{h(j)}-\left(1-y_{i h}\right) M \\
(i=1,2, \ldots, n ; h \in G) \\
\sum_{j=1}^{m} w_{j}^{*} \bar{v}_{i(j)} \leq \sum_{j=1}^{m} w_{j}^{*} \bar{v}_{h(j)}+y_{i h} M \\
(i=1,2, \ldots, n ; h \in G)
\end{array}\right.
$$

then, $r_{w^{*}}\left(x_{h}\right)=r^{*}\left(x_{h}\right),(h \in G)$.

The proof of Lemma 2 is provided in Appendix A.

Based on Lemmas 1 and 2, we obtain the following theorem.

Theorem 1: By introducing the transformed decision variables: $z_{j q}^{k}=0$ or $1, \sum_{q=1}^{3} z_{j q}^{k}=1, \quad(k=$ $1,2, \ldots, l ; q=1,2,3 ; j=1,2, \ldots, m)$, we have the following.

1) If $F$ is a WA operator, the MCSWM [model (14)] can be transformed into the mixed 0-1 linear programming

$$
\begin{aligned}
& \int \min \sum_{k=1}^{l} \sum_{j=1}^{m} f_{k}\left[\left(I_{j}^{k,-}-\overline{w_{j}^{k}}\right) z_{j 1}^{k}+\left(\overline{w_{j}^{k}}-I_{j}^{k,+}\right) z_{j 3}^{k}\right] \\
& \sum_{j=1}^{m} w_{j} \bar{v}_{i j}>\sum_{j=1}^{m} w_{j} \bar{v}_{h j}-\left(1-y_{i h}\right) M \\
& (i=1,2, \ldots, n ; h \in G) \\
& \sum_{j=1}^{m} w_{j} \bar{v}_{i j} \leq \sum_{j=1}^{m} w_{j} \bar{v}_{h j}+y_{i h} M \\
& (i=1,2, \ldots, n ; h \in G) \\
& y_{i h}=1 \text { or } 0,(i=1,2, \ldots, n ; h \in G) \\
& \sum_{i=1}^{n} y_{i h}+1=r^{*}\left(x_{h}\right),(h \in G) \\
& w_{j}=\frac{\sum_{k=1}^{l} \overline{w_{j}^{k}}}{l},(j=1,2, \ldots, m) \\
& \sum_{j=1}^{m} \overline{w_{j}^{k}}=1,(k=1,2, \ldots, l) \\
& 0 \leq \overline{w_{j}^{k}} \leq 1,(k=1,2, \ldots, l ; j=1,2, \ldots, m) \\
& w_{j}^{k}=\left[I_{j}^{k,-}, I_{j}^{k,+}\right] \\
& (k=1,2, \ldots, l ; j=1,2, \ldots, m) \\
& \overline{w_{j}^{k}}-I_{j}^{k,-} \leq 0+\left(1-z_{j 1}^{k}\right) M \\
& (k=1,2, \ldots, l ; j=1,2, \ldots, m) \\
& \overline{w_{j}^{k}} \geq 0-\left(1-z_{j 1}^{k}\right) M \\
& (k=1,2, \ldots, l ; j=1,2, \ldots, m) \\
& \overline{w_{j}^{k}}-I_{j}^{k,-}>0+\left(1-z_{j 2}^{k}\right) M \\
& (k=1,2 \ldots, l ; j=1,2, \ldots, m) \\
& \overline{w_{j}^{k}}-I_{j}^{k,+} \leq 0-\left(1-z_{j 2}^{k}\right) M \\
& (k=1,2 \ldots, l ; j=1,2, \ldots, m) \\
& \overline{w_{j}^{k}}-I_{j}^{k,+}>0+\left(1-z_{j 3}^{k}\right) M \\
& (k=1,2 \ldots, l ; j=1,2, \ldots, m) \\
& \overline{w_{j}^{k}}-1 \leq 0-\left(1-z_{j 3}^{k}\right) M \\
& (k=1,2 \ldots, l ; j=1,2, \ldots, m) \\
& z_{j 1}^{k}+z_{j 2}^{k}+z_{j 3}^{k}=1 \\
& (k=1,2 \ldots, l ; j=1,2, \ldots, m) \\
& \begin{array}{c}
z_{j q}^{k}=0 \text { or } 1,(q=1,2,3 ; k=1,2, \ldots, l \\
j=1,2, \ldots, m) .
\end{array}
\end{aligned}
$$

2) In (26)-(42) above, substitute constraints (27) and (28) into constraints

$$
\left\{\begin{aligned}
\sum_{j=1}^{m} w_{j} \bar{v}_{i(j)} & >\sum_{j=1}^{m} w_{j} \bar{v}_{h(j)}-\left(1-y_{i h}\right) M \\
& (i=1,2, \ldots, n ; h \in G) \\
\sum_{j=1}^{m} w_{j} \bar{v}_{i(j)} & \leq \sum_{j=1}^{m} w_{j} \bar{v}_{h(j)}+y_{i h} M \\
& (i=1,2, \ldots, n ; h \in G)
\end{aligned}\right.
$$

If $F$ is an OWA operator, the MCSWM [model (14)]can be transformed into the mixed 0-1 linear programming model (26), (29)-(44).

The proof of Theorem 1 is provided in Appendix A.

In this paper, denote models (26)-(42) as $P_{1}$, and models (26), (29)-(44) as $P_{2}$. In both $P_{1}$ and $P_{2}$ models, $\overline{w_{j}^{k}}(k=$ $1,2, \ldots, l ; j=1,2, \ldots, m) ; y_{i h},(i=1,2, \ldots, n ; h \in G)$; $z_{j q}^{k},(q=1,2,3 ; j=1,2, \ldots, m)$ are the decision variables.

The WA and OWA are two very popular aggregation operators used in MADM problems. Compared with the WA operator, the OWA is often be used to defend against 
the strategic manipulation. For example, in Olympic gymnastics competitions, the referees score the gymnasts. Then, the maximum and the minimum scores are deleted, and the arithmetic average of other scores is used as the collective opinion of the referees. This procedure involves the use of the OWA with the weight vector $\mathbf{w}=$ $(0,1 /(m-2), \ldots, 1 /(m-2), 0)^{T}$.

Based on Theorem 1, we can obtain the optimal solution to the MCSWM via mixed 0-1 linear programming. Clearly, if the optimal solution to the MCSWM exists, a manipulator can set a strategic weight vector to obtain his/her desired ranking of the alternatives $\left\{r^{*}\left(x_{h \in G}\right) \mid G \subseteq\{1,2, \ldots, n\}\right\}$. Otherwise, it is not possible to strategically manipulate the attribute weights to achieve his/her goal.

\section{Some Desired Properties for Models $P_{1}$ and $P_{2}$}

In this section, we present some desired properties of the MCSWM. In order to make the proposed properties easy to understand, we first introduce the ranking range of an alternative.

In an MADM problem, let $W=\left\{\left(w_{1}, w_{2}, \ldots, w_{m}\right)^{T}\right.$ $\left.\sum_{j=1}^{m} w_{j}=1,0 \leq w_{j} \leq 1\right\}$ be the set of attribute weights without any constraint; $\bar{r}_{\boldsymbol{w} \in W}\left(x_{h}\right)=\min _{\boldsymbol{w} \in W} r_{w}\left(x_{h}\right)$ the best ranking of alternative $x_{h}$ under $W$; and $\bar{r}_{w \in W}\left(x_{h}\right)=\max _{w \in W} r_{w}\left(x_{h}\right)$ the worst ranking of alternative $x_{h}$ under $W$. Then, $R_{w \in W}\left(x_{h}\right)=$ $\left[\underline{r}_{w \in W}\left(x_{h}\right), \bar{r}_{w \in W}\left(x_{h}\right)\right]$ is called the ranking range of alternative $x_{h}$ under the set of attribute weights $W$.

Let $S=\left\{\left(w_{1}, w_{2}, \ldots, w_{m}\right)^{T} \mid w_{j}=\left(\left[\sum_{k=1}^{l} \overline{w_{j}^{k}}\right] / l\right), 0 \leq \overline{w_{j}^{k}} \leq\right.$ $\left.1, \sum_{j=1}^{m} \overline{w_{j}^{k}}=1, \overline{w_{j}^{k}} \in\left[I_{j}^{k,-}, I_{j}^{k,+}\right]\right\}(j=1,2, \ldots, m ; k=$ $1,2, \ldots, l)$ be the set of interval attribute weights; $\bar{r}_{w \in S}\left(x_{h}\right)=$ $\min r_{w}\left(x_{h}\right)$ best ranking of alternative $x_{h}$ under $S$; and

$\underline{r}_{\boldsymbol{w} \in S}\left(x_{h}\right)=\max _{\boldsymbol{w} \in S} r_{\boldsymbol{w}}\left(x_{h}\right)$ the worst ranking of alternative $x_{h}$ under $S$. Then, $R_{w \in S}\left(x_{h}\right)=\left[\underline{r}_{w \in S}\left(x_{h}\right), \bar{r}_{w \in S}\left(x_{h}\right)\right]$ is called the ranking range of alternative $x_{h}$ under the set of interval attribute weights $S$.

Specifically, when the WA operator $F$, as per (3), is used to compute the decision evaluation value, let $R_{w \in W}^{\mathrm{WA}}\left(x_{h}\right)=$ $\left[\underline{r}_{w \in W}^{\mathrm{WA}}\left(x_{h}\right), \bar{r}_{w \in W}^{\mathrm{WA}}\left(x_{h}\right)\right]$ and $R_{w \in S}^{\mathrm{WA}}\left(x_{i}\right)=\left[\underline{r}_{w \in S}^{\mathrm{WA}}\left(x_{i}\right), \bar{r} \mathrm{WA} \in S\left(x_{i}\right)\right]$ be the ranking range of alternative $x_{h}$ under $W$ and $S$, respectively. When OWA operator $F$, as per (4), is used to compute the decision evaluation value, let $R_{w \in W}^{\mathrm{OWA}}\left(x_{h}\right)=$ $\left[\underline{r}_{w \in W}^{\mathrm{OWA}}\left(x_{h}\right), \bar{r}_{w \in W}^{\mathrm{OWA}}\left(x_{h}\right)\right]$ and $R_{w \in S}^{\mathrm{OWA}}\left(x_{h}\right)=\left[\underline{r}_{w \in S}^{\mathrm{OWA}}\left(x_{h}\right), \bar{r}_{w \in S}^{\mathrm{OWA}}\left(x_{h}\right)\right]$ be the ranking range of alternative $x_{h}$ under $W$ and $S$, respectively.

Then, the following three desired properties to manipulate the attribute weights are presented as Properties 1-3.

Property 1: For a desired ranking $\left\{r^{*}\left(x_{h \in G}\right) \mid G \subseteq\right.$ $\{1,2, \ldots, n\}\}$ and $|G| \geq 1$, we have the following:

1) If the objective value of $P_{1}$ is zero, then $r^{*}\left(x_{h}\right) \in$ $\left[\underline{r}_{w \in S}^{\mathrm{WA}}\left(x_{h}\right), \bar{r}_{w \in S}^{\mathrm{WA}}\left(x_{h}\right)\right], \forall h \in G$.

2) If the objective value of $P_{2}$ is zero, then $r^{*}\left(x_{h}\right) \in$ $\left[\underline{r}_{w \in S}^{\mathrm{OWA}}\left(x_{h}\right), \bar{r}_{\boldsymbol{w} \in S}^{\mathrm{OWA}}\left(x_{h}\right)\right], \forall h \in G$.

The proof of Property 1 is provided in Appendix A.

Property 1 provides the necessary condition that make possible for a manipulator to manipulate a strategic attribute weight with zero cost to obtain a desired ranking of alternatives under the WA and OWA operators, respectively.

Property 2: For a desired ranking $\left\{r^{*}\left(x_{h \in G}\right) \mid G \subseteq\right.$ $\{1,2, \ldots, n\}\}$ and $|G|=1$, we have the following:

1) The objective value of $P_{1}$ is zero if and only if $r^{*}\left(x_{h \in G}\right) \in\left[\underline{r}_{w \in S}^{\mathrm{WA}}\left(x_{h}\right), \bar{r}_{w \in S}^{\mathrm{WA}}\left(x_{h}\right)\right]$.

2) The objective value of $P_{2}$ is zero if and only if $r^{*}\left(x_{h \in G}\right) \in\left[\underline{r}_{w \in S}^{\mathrm{OWA}}\left(x_{h}\right), \bar{r}_{w \in S}^{\mathrm{OWA}}\left(x_{h}\right)\right]$.

The proof of Property 2 is provided in Appendix A.

Property 2 provides the necessary and sufficient condition for a manipulator to manipulate a strategic attribute weight with zero cost to obtain any desired ranking of one alternative under the WA and OWA operators, respectively. Notably, for the case that $|G| \geq 2$, we only can obtain a necessary condition for the zero cost manipulation (see Property 1).

Property 3: For a desired ranking $\left\{r^{*}\left(x_{h \in G}\right) \mid G \subseteq\right.$ $\{1,2, \ldots, n\}\}$ and $|G| \geq 1$, we have the following:

1) The solution of model $P_{1}$ does not exist if it satisfies the condition $\exists h \in G, r^{*}\left(x_{h}\right) \notin$ $\left[\underline{r}_{w \in W}^{\mathrm{WA}}\left(x_{h}\right), \bar{r}_{\boldsymbol{w} \in W}^{\mathrm{WA}}\left(x_{h}\right)\right]$.

2) The solution of model $P_{2}$ does not exist if it satisfies the condition $\exists h \in G, r^{*}\left(x_{h}\right) \notin$ $\left[\underline{r}_{w \in W}^{\mathrm{OWA}}\left(x_{h}\right), \bar{r}_{\boldsymbol{w} \in W}^{\mathrm{OWA}}\left(x_{h}\right)\right]$.

The proof of Property 3 is provided in Appendix A.

Property 3 provides the condition under which a manipulator cannot manipulate a strategic weight vector under any cost to obtain his/her desired ranking.

\section{NUMERICAL ANALYSIS AND SIMULATION EXPERIMENTS}

In this section, we present an example with real data (provided in Appendix B) from the Academic Ranking of World Universities (ARWU; http://www.arwu.org/) [34] and several simulation experiments to show the validity and desired properties of the proposed MCSWM model.

\section{A. Numerical Analysis}

Let 50 Universities taken from ARWU be the set of alternatives $\left\{x_{1}, x_{2}, \ldots, x_{50}\right\}$, which will be ranked using the following set of six attributes $\left\{a_{1}, a_{2}, \ldots, a_{6}\right\}$.

$a_{1}$ : Quality of Education (Alumni: Alumni of an institution winning Nobel Prizes and Fields Medals).

$a_{2}$ : Quality of Faculty 1 (Award: Staff of an institution winning Nobel Prizes and Fields Medals).

$a_{3}$ : Quality of Faculty 2 (HiCi: Highly Cited researchers in 21 broad subject categories).

$a_{4}$ : Papers published in Nature and Science (N\&S).

$a_{5}$ : Papers indexed in Science Citation Index-expanded and Social Science Citation Index (PUB).

$a_{6}$ : Per capita academic performance of an institution (PCP).

First, we transform the data for the 50 universities regarding the set of attributes above into a normalized decision matrix $\bar{V}=\left[\bar{v}_{i j}\right]_{50 \times 6}$. Let $E=\left\{e_{1}, e_{2}, e_{3}\right\}$ be a set of three experts. Let $\mathbf{w}^{1}=\left(w_{1}^{1}, w_{2}^{1}, \ldots, w_{6}^{1}\right)^{T}$, where $w_{1}^{1}=[0.1,0.3]$, and $w_{j}^{1}=$ $[0,1], j=2,3,4,5,6$ are the interval attribute weights of 
expert $e_{1} ; \mathbf{w}^{2}=\left(w_{1}^{2}, w_{2}^{2}, \ldots, w_{6}^{2}\right)^{T}$, where $w_{2}^{2}=[0.2,0.6]$, and $w_{j}^{2}=[0,1], j=1,3,4,5,6$ are the interval attribute weights of expert $e_{2}$; and $\mathbf{w}^{3}=\left(w_{1}^{3}, w_{2}^{3} \ldots, w_{6}^{3}\right)^{T}$, where $w_{4}^{3}=$ $[0.4,0.8], w_{5}^{3}=[0.2,0.9]$, and $w_{j}^{3}=[0,1], j=1,2,3,6$ are the interval attribute weights of expert $e_{3}$.

Without loss of generality, let $f_{k}=1,(k=1,2,3)$, be the unit cost of revising decision maker's original attribute weights. In the following, we assume that an expert wants to manipulate the alternative $x_{h}$, and his/her desired ranking for such alternative is $r^{*}$. Then, based on models $P_{1}$ and $P_{2}$, the manipulator can strategically set an attribute weight vector $w^{*}$ with minimum cost $C^{*}$, to obtain his/her desired goal of ranking.

1) Let $x_{3}$ be the manipulated alternative and $r^{*}\left(x_{3}\right)=$ 3 the corresponding desired ranking. If $F$ is the WA operator, then this is possible as $P_{1}$ results in the following strategic attribute weight vector $\mathbf{w}^{*}=$ $(0.367,0.067,0.033,0.133,0.4,0)$ with minimum cost $C^{*}=0$.

2) Let $x_{20}$ be the manipulated alternative, and $r^{*}\left(x_{20}\right)=$ 15 the corresponding desired ranking. If $F$ is the OWA operator, then this is possible as $P_{2}$ results in the following strategic weight vector $\mathbf{w}^{*}=$ $(0.421,0.375,0,0,0.011,0.194)$ with minimum cost $C^{*}=0.57$.

3) Let $\left\{x_{8}, x_{13}, x_{14}, x_{15}\right\}$ be the manipulated alternatives, and $r^{*}=\{46,23,24,13\}$ their corresponding desired ranking. If $F$ is WA operator, then this is possible with $P_{1}$ resulting in the following strategic weight vector $\mathbf{w}^{*}=(0.064,0.081,0,0.006,0.849,0)$ with minimum cost $C^{*}=0.382$.

4) Let $\left\{x_{9}, x_{10}, x_{11}, x_{12}\right\}$ be the manipulated alternatives, and $r^{*}=\{19,7,27,16\}$ their desired ranking. If $F$ is OWA operator, then because there is no solution to $P_{2}$, the manipulator will be unable to strategically set an attribute weight vector to achieve the desired ranking.

Table I shows a strategic weight vector $w^{*}$ with its corresponding minimum cost $C^{*}$ for different manipulated alternative(s) $x^{*}$ to achieve a desired ranking $r^{*}$.

From Table I, it can be noticed that in some cases the manipulator incurred zero cost $\left(C^{*}=0\right)$ to set a strategic weight vector to obtain his/her goal. On the other hand, in some other cases the manipulator is unable to set a strategic weight vector under any cost. In the following, we will verify the validity of the conditions presented in Properties 1-3. Table II shows the ranking ranges $R_{w \in W}^{\mathrm{WA}}, R_{w \in W}^{\mathrm{OWA}}, R_{w \in S}^{\mathrm{WA}}$, and $R_{w \in S}^{\mathrm{OWA}}$ for the 50 universities.

Based on the data from Tables I and II, we find the results to be consistent with Properties 1-3.

\section{B. Simulation Experiments}

In this section, we present simulation experiments to analyze the effect the interval attribute weights and the unit cost have on the MCSWM.

1) Effect of Interval Attribute Weights: First, we consider the constraints for the attribute weights. Let $S_{j}=$ $\left\{\left(w_{1}, \ldots, w_{j}, \ldots, w_{m}\right)^{T} \mid w_{i} \in\left[I_{i}^{-}, I_{i}^{+}\right]\right\}(j=1,2, \ldots, m)$ be
TABLE I

Strategic Weight Vector $w^{*}$ With Minimum Cost $C^{*}$ For DifFerent MANipulated Alternative(s) $x^{*}$ AND DESIRED RANKING $r^{*}$

\begin{tabular}{|c|c|c|c|}
\hline \multicolumn{4}{|c|}{ WA } \\
\hline $\begin{array}{l}\text { Manipulated } \\
\text { alternative(s) }\end{array}$ & $r^{*}$ & $\mathbf{w}^{*}$ & $C^{*}$ \\
\hline$x_{3}$ & 3 & $(0.37,0.07,0.03,0.13,0.4,0)$ & 0 \\
\hline$x_{6}$ & 10 & $(0.03,0.06,0,0.13,0.77,0.03)$ & 0.019 \\
\hline$x_{20}$ & 9 & No solution & $\sim$ \\
\hline \multirow[t]{2}{*}{$x_{8}, x_{13}, x_{14}, x_{15}$} & $\{2,6,10,12\}$ & No solution & $\sim$ \\
\hline & $\{46,23,24,13\}$ & $(0.06,0.08,0,0.01,0.85,0)$ & 0.382 \\
\hline \multirow{2}{*}{$x_{9}, x_{10}, x_{11}, x_{12}$} & $\{6,7,8,9\}$ & No solution & $\sim$ \\
\hline & $\{6,8,9,10\}$ & $(0.12,0.2,0,0.13,0.54,0)$ & 0 \\
\hline \multirow{2}{*}{$x_{20}, x_{23}, x_{25}, x_{27}$} & $\{13,3,16,4\}$ & $(0,0,0.17,0.01,0.82,0)$ & 0.667 \\
\hline & $\{10,2,15,3\}$ & No solution & $\sim$ \\
\hline \multicolumn{4}{|c|}{ OWA } \\
\hline $\begin{array}{l}\text { Manipulated } \\
\text { alternative(s) }\end{array}$ & $r^{*}$ & $\mathbf{w}^{*}$ & $C^{*}$ \\
\hline$x_{3}$ & 8 & $(0.98,0.02,0,0,0,0)$ & 1.37 \\
\hline$x_{6}$ & 6 & $(0.37,0.13,0.3,0.13,0.07,0)$ & 0 \\
\hline$x_{20}$ & 15 & $(0.42,0.38,0,0,0.01,0.19)$ & 0.57 \\
\hline \multirow[t]{2}{*}{$x_{8}, x_{13}, x_{14}, x_{15}$} & $\{10,11,12,13\}$ & No solution & $\sim$ \\
\hline & $\{6,12,13,14\}$ & $(0.1,0.07,0.63,0.01,03,0.07)$ & 0.357 \\
\hline \multirow{2}{*}{$x_{9}, x_{10}, x_{11}, x_{12}$} & $\{8,9,10,11\}$ & $(0.03,0.83,0,0.13,0.01,0)$ & 0.198 \\
\hline & $\{19,7,27,16\}$ & No solution & $\sim$ \\
\hline \multirow{2}{*}{$x_{20}, x_{23}, x_{25}, x_{27}$} & $\{17,9,21,10\}$ & $(0.03,0.04,0.13,0.13,0.6,0.05)$ & 0.069 \\
\hline & $\{24,45,46,47\}$ & No solution & $\sim$ \\
\hline
\end{tabular}

a set of interval attribute weights, where $\left[I_{i}^{-}, I_{i}^{+}\right] \subset[0,1]$ $(i \leq j)$ and $\left[I_{i}^{-}, I_{i}^{+}\right]=[0,1](i>j)$. In the other words, set $S_{j}$ constraints only the weight of an attribute $a_{i}$ with $i \leq j$. In Simulation Experiment I below, set $S_{j}$ is randomly generated, and thus the bigger the value $j$ the more constraints on attribute weights there are, in the sense of average cases.

Let $r^{*}\left(x_{h}\right)$ be the manipulator's desired ranking of the alternative $x_{h}$, and $f_{k}(k=1,2, \ldots, l)$ the unit cost to revise the expert $e_{k}$ 's original interval attribute weights. Let $F_{S_{j}}^{\mathrm{WA}}\left(x_{h}\right)$ and $F_{S_{j}}^{\mathrm{OWA}}\left(x_{h}\right)$ be the minimum cost to find out a strategic weight vector from the set $S_{j}$ to obtain the manipulator's desired goal ranking of alternative $x_{h}$ under the WA and the OWA operators, respectively.

Next, we design Simulation Experiment I to analyze the effect of interval attribute weights on the minimum cost to manipulate a strategic weight vector. Without loss of 
TABLE II

RANKING RANGES $R_{w \in W}^{\mathrm{WA}}, R_{\boldsymbol{w} \in W}^{\mathrm{OWA}}, R_{\boldsymbol{w} \in S}^{\mathrm{WA}}$, AND $R_{\boldsymbol{w} \in S}^{\mathrm{OWA}}$ FOR 50 Universities

\begin{tabular}{|c|c|c|c|c|c|c|}
\hline$x_{i}$ & $x_{1}$ & $x_{2}$ & $x_{3}$ & $x_{4}$ & $x_{5}$ & $x_{6}$ \\
\hline$R_{w \in W}^{W A}$ & {$[1,2]$} & {$[2,11]$} & {$[2,13]$} & {$[2,14]$} & {$[2,25]$} & {$[2,47]$} \\
\hline$R_{w \in W}^{O W A}$ & {$[1,2]$} & {$[2,7]$} & {$[2,9]$} & {$[2,5]$} & {$[2,7]$} & {$[2,9]$} \\
\hline$R_{w \in S}^{W A}$ & {$[1,1]$} & {$[2,4]$} & {$[3,5]$} & {$[2,5]$} & {$[2,5]$} & {$[6,9]$} \\
\hline$R_{w \in S}^{O W A}$ & {$[1,1]$} & {$[2,2]$} & {$[4,5]$} & {$[4,5]$} & {$[3,3]$} & {$[6,6]$} \\
\hline$x_{i}$ & $x_{7}$ & $x_{8}$ & $x_{9}$ & $x_{10}$ & $x_{11}$ & $x_{12}$ \\
\hline$R_{w \in W}^{W A}$ & {$[2,9]$} & {$[1,47]$} & {$[4,28]$} & {$[4,42]$} & {$[5,26]$} & {$[4,27]$} \\
\hline$R_{w \in W}^{O W A}$ & {$[5,11]$} & {$[1,9]$} & {$[6,17]$} & {$[6,12]$} & {$[8,24]$} & {$[10,19]$} \\
\hline$R_{w \in S}^{W A}$ & {$[7,9]$} & {$[6,11]$} & {$[6,8]$} & {$[8,11]$} & {$[8,11]$} & {$[12,15]$} \\
\hline$R_{w \in S}^{O W A}$ & {$[8,8]$} & {$[7,7]$} & {$[9,9]$} & {$[10,10]$} & {$[11,11]$} & {$[12,12]$} \\
\hline$x_{i}$ & $x_{13}$ & $x_{14}$ & $x_{15}$ & $x_{16}$ & $x_{17}$ & $x_{18}$ \\
\hline$R_{w \in W}^{W A}$ & {$[9,28]$} & {$[5,36]$} & {$[5,36]$} & {$[7,33]$} & {$[5,30]$} & {$[9,28]$} \\
\hline$R_{w \in W}^{O W A}$ & {$[8,30]$} & {$[11,31]$} & {$[11,31]$} & {$[10,25]$} & {$[10,30]$} & {$[13,27]$} \\
\hline$R_{w \in S}^{W A}$ & {$[12,14]$} & {$[12,15]$} & {$[14,16]$} & {$[13,16]$} & {$[17,20]$} & {$[20,21]$} \\
\hline$R_{w \in S}^{O W A}$ & {$[13,13]$} & {$[14,14]$} & {$[17,22]$} & {$[15,15]$} & {$[16,20]$} & {$[16,21]$} \\
\hline$x_{i}$ & $x_{19}$ & $x_{20}$ & $x_{21}$ & $x_{22}$ & $x_{23}$ & $x_{24}$ \\
\hline$R_{w \in W}^{W A}$ & {$[8,38]$} & {$[10,43]$} & {$[11,50]$} & {$[10,46]$} & {$[3,50]$} & {$[9,46]$} \\
\hline$R_{w \in W}^{O W A}$ & {$[13,36]$} & {$[15,34]$} & {$[15,50]$} & {$[18,43]$} & {$[9,50]$} & {$[15,46]$} \\
\hline$R_{w \in S}^{W A}$ & {$[17,19]$} & {$[17,20]$} & {$[19,21]$} & {$[25,31]$} & {$[22,33]$} & {$[22,29]$} \\
\hline$R_{w \in S}^{O W A}$ & {$[16,22]$} & {$[23,25]$} & {$[16,21]$} & {$[22,23]$} & {$[16,24]$} & {$[16,23]$} \\
\hline$x_{i}$ & $x_{25}$ & $x_{26}$ & $x_{27}$ & $x_{28}$ & $x_{29}$ & $x_{30}$ \\
\hline$R_{w \in W}^{W A}$ & {$[5,49]$} & {$[8,45]$} & {$[2,48]$} & {$[15,48]$} & {$[22,50]$} & {$[9,47]$} \\
\hline$R_{w \in W}^{O W A}$ & {$[13,48]$} & {$[14,43]$} & {$[8,48]$} & {$[19,44]$} & {$[23,41]$} & {$[20,42]$} \\
\hline$R_{w \in S}^{W A}$ & {$[27,35]$} & {$[31,37]$} & {$[22,26]$} & {$[22,25]$} & {$[22,26]$} & {$[28,35]$} \\
\hline$R_{w \in S}^{O W A}$ & {$[26,34]$} & {$[24,25]$} & {$[26,34]$} & {$[26,29]$} & {$[27,31]$} & {$[26,29]$} \\
\hline$x_{i}$ & $x_{31}$ & $x_{32}$ & $x_{33}$ & $x_{34}$ & $x_{35}$ & $x_{36}$ \\
\hline$R_{w \in W}^{W A}$ & {$[17,43]$} & {$[16,50]$} & {$[16,50]$} & {$[16,49]$} & {$[22,48]$} & {$[22,49]$} \\
\hline$R_{w \in W}^{O W A}$ & {$[17,43]$} & {$[21,43]$} & {$[20,41]$} & {$[22,48]$} & {$[27,43]$} & {$[25,49]$} \\
\hline$R_{w \in S}^{W A}$ & {$[27,31]$} & {$[25,31]$} & {$[33,39]$} & {$[35,41]$} & {$[38,49]$} & {$[36,43]$} \\
\hline$R_{w \in S}^{O W A}$ & {$[29,35]$} & {$[30,34]$} & {$[32,35]$} & {$[36,40]$} & {$[36,38]$} & {$[29,34]$} \\
\hline$x_{i}$ & $x_{37}$ & $x_{38}$ & $x_{39}$ & $x_{40}$ & $x_{41}$ & $x_{42}$ \\
\hline$R_{w \in W}^{W A}$ & {$[10,50]$} & {$[21,49]$} & {$[15,50]$} & {$[13,50]$} & {$[27,49]$} & {$[15,50]$} \\
\hline$R_{w \in W}^{O W A}$ & {$[20,48]$} & {$[22,49]$} & {$[24,49]$} & {$[18,50]$} & {$[33,50]$} & {$[24,50]$} \\
\hline$R_{w \in S}^{W A}$ & {$[23,40]$} & {$[32,38]$} & {$[29,36]$} & {$[44,49]$} & {$[41,44]$} & {$[45,49]$} \\
\hline$R_{w \in S}^{O W A}$ & {$[38,43]$} & {$[27,35]$} & {$[38,42]$} & {$[41,48]$} & {$[45,48]$} & {$[36,42]$} \\
\hline$x_{i}$ & $x_{43}$ & $x_{44}$ & $x_{45}$ & $x_{46}$ & $x_{47}$ & $x_{48}$ \\
\hline$R_{w \in W}^{W A}$ & {$[22,50]$} & {$[13,50]$} & {$[28,50]$} & {$[11,50]$} & {$[21,50]$} & {$[15,50]$} \\
\hline$R_{w \in W}^{O W A}$ & {$[26,50]$} & {$[23,50]$} & {$[37,50]$} & {$[22,50]$} & {$[29,49]$} & {$[24,49]$} \\
\hline$R_{w \in S}^{W A}$ & {$[37,45]$} & {$[45,47]$} & {$[37,42]$} & {$[30,44]$} & {$[42,44]$} & {$[46,49]$} \\
\hline$R_{w \in S}^{O W A}$ & {$[40,48]$} & {$[40,44]$} & {$[47,49]$} & {$[36,42]$} & {$[46,49]$} & {$[42,44]$} \\
\hline$x_{i}$ & $x_{49}$ & $x_{50}$ & & & & \\
\hline$R_{w \in W}^{W A}$ & {$[27,50]$} & {$[30,50]$} & & & & \\
\hline$R_{w \in W}^{O W A}$ & {$[38,49]$} & {$[30,50]$} & & & & \\
\hline$R_{w \in S}^{W A}$ & {$[47,50]$} & {$[49,50]$} & & & & \\
\hline$R_{w \in S}^{O W A}$ & {$[45,49]$} & {$[50,50]$} & & & & \\
\hline
\end{tabular}

generality, we set $f_{k}=1,(k=1,2, \ldots, l)$ and set the manipulated alternative to be $x_{1}$.

Simulation Experiment I:

Input: $n, m$, and $j$.

Output: $F_{S_{j}}^{\mathrm{WA}}\left(x_{1}\right)$ and $F_{S_{j}}^{\mathrm{OWA}}\left(x_{1}\right)$.

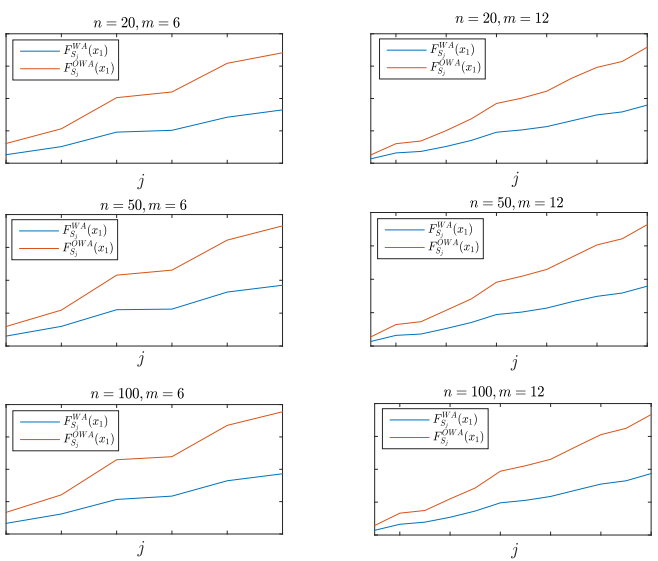

Fig. 1. Average values of $F_{S_{j}}^{\mathrm{WA}}\left(x_{1}\right)$ and $F_{S_{j}}^{\mathrm{OWA}}\left(x_{1}\right)$ under different parameters in Simulation Experiment I.

Step 1 (Generation of the Standardized Decision Matrix): Generate randomly a standardized decision matrix $\bar{V}=$ $\left[\bar{v}_{i j}\right]_{n \times m}$, where $\bar{v}_{i j} \in[0,1]$.

Step 2 (Generation of the Desired Ranking of the Alternative $\left.x_{1}\right)$ : Apply methods from Dong et al. [12] to obtain the ranking ranges of the alternative $x_{1},\left[\underline{r}_{w \in W}^{\mathrm{WA}}\left(x_{1}\right), \bar{r}_{w \in W}^{\mathrm{WA}}\left(x_{1}\right)\right]$ and $\left[\underline{r}_{w \in W}^{\mathrm{OWA}}\left(x_{1}\right), \bar{r}_{w \in W}^{\mathrm{OWA}}\left(x_{1}\right)\right]$, for the WA and the OWA operators, respectively. Let $r^{*}\left(x_{1}\right)$ be the manipulator's desired ranking of the alternative $x_{1}$. When using the WA operator, the value of $r^{*}\left(x_{1}\right)$ is randomly selected from $\left[\underline{r}_{w \in W}^{\mathrm{WA}}\left(x_{1}\right), \bar{r}_{w \in W}^{\mathrm{WA}}\left(x_{1}\right)\right]$. When using the OWA operator, the value of $r^{*}\left(x_{1}\right)$ is randomly selected from $\left[\underline{r}_{w \in W}^{\mathrm{OWA}}\left(x_{1}\right), \bar{r}_{w \in W}^{\mathrm{OWA}}\left(x_{1}\right)\right]$.

Step 3 (Generation of the Interval Attribute Weights Sets $\left.S_{j}\right)$ : Generate randomly a set of interval attribute weights $S_{j}=\left\{\left(w_{1}, \ldots, w_{j}, \ldots, w_{m}\right)^{T} \mid w_{i} \in\left[I_{i}^{-}, I_{i}^{+}\right]\right\}$; generate a random integer number $j$ from set $\{1,2, \ldots, m\}$; generate random values $I_{i}^{-}(i \leq j)$ and $I_{i}^{+}(i \leq j)$ from $[0,1)$ and $\left[I_{i}^{-}, 1\right)$, respectively, and set $\left[I_{i}^{-}, I_{i}^{+}\right]=[0,1](i>j)$. Apply models $P_{1}$ and $P_{2}$ to obtain $F_{S_{j}}^{\mathrm{WA}}\left(x_{1}\right)$ and $F_{S_{j}}^{\mathrm{OWA}}\left(x_{1}\right)$, respectively. Compute the minimum cost $F_{S_{j}}^{\mathrm{WA}}\left(x_{1}\right)$ and $F_{S_{j}}^{\mathrm{OWA}}\left(x_{1}\right)$ to find out a strategic weight vector from the set $S_{j}$ to obtain the manipulator's desired goal ranking of alternative $x_{1}$ under the WA and the OWA operators, respectively.

We set different values of $n, m$, and $j$, and run 100 times Simulation Experiment I to obtain average values of $F_{S_{j}}^{\mathrm{WA}}\left(x_{1}\right)$ and $F_{S_{j}}^{\mathrm{OWA}}\left(x_{1}\right)$, which are shown in Fig. 1 below.

Clearly, Fig. 1 shows that: 1) in all cases, the average minimum cost to set strategic weight vectors under the WA operator is smaller than that under the OWA operator and 2) the average minimum cost to set strategic weight vectors under the OWA operator increase more quickly than that under the WA operator, as the attribute weights constraints increase.

2) Effect of the Unit Cost: In Simulation Experiment II, we assumed that the unit cost to revise the original attribute weights information is the same for all experts, i.e., $f_{k}=$ $f,(k=1,2, \ldots, l)$, and respectively, set as $f=(u / 100)$ $(u=1,2, \ldots, 100)$ to study the effect of unit cost on the minimum cost to strategically manipulate the attribute weight vector in the MCSWM. Clearly, the larger the value of $u$, the higher the unit cost is. 

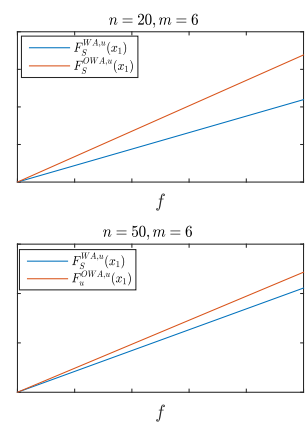

$n=100, m=6$

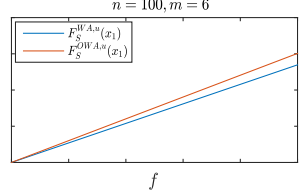

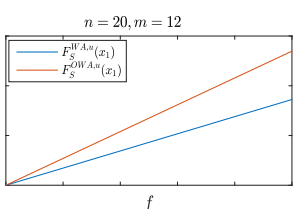

$f$

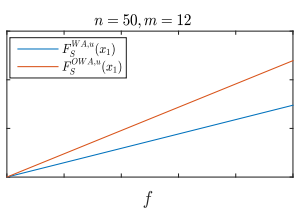

$n=100, m=12$

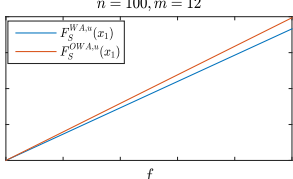

Fig. 2. Average values of $F_{S}^{\mathrm{WA}, u}\left(x_{1}\right)$ and $F_{S}^{\mathrm{OWA}, u}\left(x_{1}\right)$ under different parameters in Simulation Method II.

Let $r^{*}\left(x_{h}\right)$ be the manipulator's desired ranking of the alternative $x_{h}$. When setting $f=(u / 100)$, let $F_{S}^{\mathrm{WA}, u}\left(x_{1}\right)$ be the minimum cost to find out a strategic weight vector from a set of interval attribute weights $S$ to achieve the manipulator's desired goal ranking of alternative $x_{h}$ under the WA and the OWA operators, respectively. Without loss of generality, we set the manipulated alternative to be $x_{1}$ and the sets of interval attribute weights are generated randomly.

Simulation Experiment II:

Input: $n, m$, and $u$.

Output: $F_{S}^{\mathrm{WA}, u}\left(x_{1}\right)$ and $F_{S}^{\mathrm{OWA}, u}\left(x_{1}\right)$.

Step 1: Same as step 1 in Simulation Experiment I.

Step 2: Same as step 2 in Simulation Experiment I.

Step 3 (Generation of the Interval Attribute Weights Sets $S)$ : Generate the set of interval attribute weights $S=$ $\left\{\left(w_{1}, w_{2}, \ldots, w_{m}\right)^{T} \mid w_{j} \in\left[I_{j}^{-}, I_{j}^{+}\right], j=1,2, \ldots, m\right\}$ by randomly selecting $I_{j}^{-}$and $I_{j}^{+}$from $[0,1)$ and $\left[I_{j}^{-}, 1\right)$, respectively.

Step 4 (Calculation of the Minimum Cost $F_{S}^{\mathrm{WA}, u}\left(x_{1}\right)$ and $\left.F_{S}^{\text {OWA }, u}\left(x_{1}\right)\right)$ : Set $f_{k}=f,(k=1,2, \ldots, l)$ and $f=\frac{u}{100}$. Apply models $P_{1}$ and $P_{2}$ to obtain $F_{S}^{\mathrm{WA}, u}\left(x_{1}\right)$ and $F_{S}^{\mathrm{OWA}, u}\left(x_{1}\right)$, respectively. Compute the minimum cost $F_{S}^{\mathrm{WA}, u}\left(x_{1}\right)$ and $F_{S}^{\mathrm{OWA}, u}\left(x_{1}\right)$ to find out a strategic weight vector from the set $S$ to obtain the manipulator's desired goal ranking of alternative $x_{1}$ under the WA and the OWA operators, respectively.

We set different values of $n, m$, and $u$, and run 100 times Simulation Experiment II to obtain average values of $F_{S}^{\mathrm{WA}, u}\left(x_{1}\right)$ and $F_{S}^{\mathrm{OWA}, u}\left(x_{1}\right)$, which are depicted in Fig. 2.

As with Simulation Method I, it is evident from Fig. 2 that 1) in all cases, the average minimum cost to set strategic weight vectors under the OWA operator is larger than that under the WA operator, and 2) as the unit cost increases, the average minimum cost to set strategic weight vectors under the OWA operator increases more quickly than that under the WA operator.

Simulation Methods I and II both show a better performance of the OWA operator than the WA operator in defending against the strategic weight manipulation of the MADM problems because of the higher associated minimum cost.
Furthermore, as the attribute weights constraints and the unit cost increase, the performance of the OWA operator as a defense mechanism against the strategic weight manipulation increases faster than if the WA operator were used instead.

Consequently, it can be concluded that the OWA operator provides a better defense mechanism than the WA operator against multiple attribute strategic weight manipulation with interval attribute weights information.

\section{CONCLUSION}

This paper focuses on the strategic weight manipulation with minimum cost to obtain a desired ranking of alternatives, in a group decision context with interval attribute weights information. The existing approaches to set attribute weights have been investigated intensively, however, in these approaches decision makers are assumed to be honest aiming to obtain "best" attribute weights to get a ranking of alternatives. This paper follows the new assumption presented in [12] that the decision makers are not honest to strategically set attribute weights to obtain their desired ranking of the alternatives. The main contributions presented in this paper are as follows.

1) The strategic weight manipulation issue in [12] was investigated in an individual decision making context with no constraints on the attribute. In this paper, we present the MCSWM model in a group decision context with interval attribute weights information.

2) We discuss the conditions based on the ranking range under interval attribute weights information for a) the existence of a weight vector to be set strategically to achieve the manipulator's desired ranking and b) zero cost for the manipulation.

3) We present detailed simulation experiments to reveal the effects of the attribute weights information and the unit cost on the minimum cost to manipulate strategic weights in a group context.

Meanwhile, we argue that it will be an interesting future research topic to investigate multiple attribute strategic weight manipulation in a consensus-reaching context [14], [49], [50] and the presence of trust relationship [39], [40].

\section{APPENDIX A PROOFS}

Proof of Lemma 1: 1) Substitute $y_{i h}=1$ into constraints (15) and (16), then $\sum_{j=1}^{m} w_{j}^{*} \bar{v}_{i j}>\sum_{j=1}^{m} w_{j}^{*} \bar{v}_{h j}$ and $\sum_{j=1}^{m} w_{j}^{*} \bar{v}_{i j} \leq \sum_{j=1}^{m} w_{j}^{*} \bar{v}_{h j}+1 \cdot M,(i=1,2, \ldots, n ; h \in G)$ can be obtained. According to the result (1) in Section III-A, $x_{i} \succ x_{h}(h \in G)$ can be guaranteed. If $y_{i h}=0$, then $\sum_{j=1}^{m} w_{j}^{*} \bar{v}_{i j} \leq \sum_{j=1}^{m} w_{j}^{*} \bar{v}_{h j}$ and $\sum_{j=1}^{m} w_{j}^{*} \bar{v}_{i j}>\sum_{j=1}^{m} w_{j}^{*} \bar{v}_{h j}-$ $1 \cdot M,(i=1,2, \ldots, n ; h \in G)$. According to result (2) in Section III-A, $x_{i \sim} \prec x_{h}(h \in G)$ can be guaranteed. Due to $\mathbf{w}^{*}=\left(w_{1}^{*}, w_{2}^{*}, \ldots, w_{m}^{*}\right)$ verifying constraints (15)-(23), the strategic weight vector $\mathbf{w}^{*}$ can be obtained by revising the decision maker's original attribute weights, so the distance $d\left(\overline{w_{j}^{*, k}}, w_{j}^{k}\right)$ should be given. Based on the non-negative property of distance functions, we can obtain different distance 
formula for the different ranges of $\mathbf{w}^{*}$, i.e.,

$$
d\left(\overline{w_{j}^{*, k}}, w_{j}^{k}\right)= \begin{cases}I_{j}^{k,-}-\overline{w_{j}^{*, k}}, & 0 \leq \overline{w_{j}^{*, k}} \leq I_{j}^{k,-} \\ 0, & I_{j}^{k,-}<\overline{w_{j}^{*, k}} \leq I_{j}^{k,+} \\ \overline{w_{j}^{*, k}}-I_{j}^{k,+}, & I_{j}^{k,+}<\overline{w_{j}^{*, k}} \leq 1 .\end{cases}
$$

Finally, the constraint condition $\sum_{i=1}^{n} y_{i h}+1=$ $r^{*}\left(x_{h}\right),(h \in G)$ can guarantee $r_{w^{*}}\left(x_{h}\right)=r^{*}\left(x_{h}\right),,(h \in G)$.

This completes the proof of Lemma 1.

Proof of Lemma 2: Substitute the WA operator $\sum_{j=1}^{m} w_{j}^{*} \bar{v}_{i j}>\sum_{j=1}^{m} w_{j}^{*} \bar{v}_{h j}-\left(1-y_{i h}\right) M$ and $\sum_{j=1}^{m} w_{j}^{*} \bar{v}_{i j} \leq \sum_{j=1}^{m} w_{j}^{*} \bar{v}_{h j}+y_{i h} M,(i=1,2, \ldots, n ; h \in G)$ into the OWA operator $\sum_{j=1}^{m} w_{j}^{*} \bar{v}_{i(j)}>\sum_{j=1}^{m} w_{j}^{*} \bar{v}_{h(j)}-\left(1-y_{i h}\right) M$ and $\sum_{j=1}^{m} w_{j}^{*} \bar{v}_{i(j)} \leq \sum_{j=1}^{m} w_{j}^{*} \bar{v}_{h(j)}+y_{i h} M,(i=1,2, \ldots, n$; $h \in G)$ in proof of Lemma 1 and conclude that $r_{w^{*}}\left(x_{h}\right)=r^{*}\left(x_{h}\right),(h \in G)$.

This completes the proof of Lemma 2.

Proof of Theorem 1: Introduce the following transformed decision variables $z_{j q}^{k}$, with $z_{j q}^{k}=0$ or $1(q=$ $1,2,3), \sum_{q=1}^{3} z_{j q}^{k}=1,(k=1,2, \ldots, l ; j=1,2, \ldots, m)$. Because $w_{j}^{k}=\left[I_{j}^{k,-}, I_{j}^{k,+}\right]$

$$
d\left(w_{j}^{k}, \overline{w_{j}^{k}}\right)= \begin{cases}I_{j}^{k,-}-\overline{w_{j}^{k}}, & 0 \leq \overline{w_{j}^{k}}<I_{j}^{k,-} \\ 0, & I_{j}^{k,-} \leq \overline{w_{j}^{k}} \leq I_{j}^{k,+} \\ \overline{w_{j}^{k}}-I_{j}^{k,+}, & I_{j}^{k,+}<\overline{w_{j}^{k}} \leq 1\end{cases}
$$

then, the mix 0-1 formulas.

$$
\overline{w_{j}^{k}}-I_{j}^{k,-} \leq 0+\left(1-z_{j 1}^{k}\right) M \text { and } \overline{w_{j}^{k}} \geq 0-\left(1-z_{j 1}^{k}\right) M \text { guarantee }
$$
$0 \leq \overline{w_{j}^{k}}<I_{j}^{k,-}$.

$\overline{w_{j}^{k}}-I_{j}^{k,-}>0+\left(1-z_{j 2}^{k}\right) M$ and $\overline{w_{j}^{k}}-I_{j}^{k,+} \leq 0-\left(1-z_{j 2}^{k}\right) M$ guarantee $I_{j}^{k,-} \leq \overline{w_{j}^{k}} \leq I_{j}^{k,+}$.

$\overline{w_{j}^{k}}-I_{j}^{k,+}>\left(1-z_{j 3}^{k}\right) M$ and $\overline{w_{j}^{k}}-1 \leq 0-\left(1-z_{j 3}^{k}\right) M$ guarantee $I_{j}^{k,+}<\overline{w_{j}^{k}} \leq 1$.

Then, we have $d\left(w_{j}^{k}, \overline{w_{j}^{k}}\right)=\left(I_{j}^{k,-}-\overline{w_{j}^{k}}\right) z_{j 1}^{k}+\left(\overline{w_{j}^{k}}-I_{j}^{k,+}\right) z_{j 3}^{k}$.

According to Lemmas 1 and 2, plug models (15)-(23) and (17)-(25) into model (14) and transform the optimization models into the mixed 0-1 linear programming models (26)-(42) and (26), (28)-(44), respectively.

This completes the proof of Theorem 1 .

Proof of Property 1: Assuming that $\forall h \in G, r^{*}\left(x_{h}\right) \in$ $\left[\underline{r}_{w \in S}^{\mathrm{WA}}\left(x_{h}\right), \bar{r}_{w \in S}^{\mathrm{WA}}\left(x_{h}\right)\right]$, the objective value of $P_{1}$ is nonzero, which means the manipulator must take some cost to revise the decision maker's original attribute weights. However, the condition $r^{*}\left(x_{h}\right) \in\left[\underline{r}_{w \in S}^{\mathrm{WA}}\left(x_{h}\right), \bar{r}_{w \in S}^{\mathrm{WA}}\left(x_{h}\right)\right]$ means the manipulator can obtain his/her ranking in the ranking range under the original interval attribute weights information. The above two results are in contradiction.

This completes the proof of Property 1.

Proof of Property 2 (Sufficiency): When $|G|=1$, the number of alternatives which are associated with the desired ranking by the manipulator is only one. Then, if $r^{*}\left(x_{h \in G}\right) \in$ $\left[\underline{r}_{w \in S}^{\mathrm{WA}}\left(x_{h}\right), \bar{r}_{w \in S}^{\mathrm{WA}}\left(x_{h}\right)\right]$, the manipulator can successfully obtain his/her ranking in the ranking range under the original interval attribute weights information, it is evident that the objective value of $P_{1}$ is zero.

\begin{tabular}{|c|c|c|c|c|c|c|}
\hline$x_{i}$ & $v_{i 1}$ & $v_{i 2}$ & $v_{i 3}$ & $v_{i 4}$ & $v_{i 5}$ & $v_{i 6}$ \\
\hline 1 & 100 & 100 & 100 & 100 & 100 & 79.2 \\
\hline 2 & 42.9 & 89.6 & 80.1 & 73.6 & 73.1 & 55.8 \\
\hline 3 & 65.1 & 79.4 & 64.9 & 68.7 & 68.4 & 59 \\
\hline 4 & 78.3 & 96.6 & 51.3 & 56.7 & 67.8 & 58.5 \\
\hline 5 & 69.4 & 80.7 & 55.3 & 71.7 & 61.7 & 69.7 \\
\hline 6 & 53.3 & 98 & 51.3 & 47.2 & 42.9 & 74.4 \\
\hline 7 & 49.7 & 54.9 & 56.2 & 55 & 74.5 & 46.1 \\
\hline 8 & 51 & 66.7 & 39.7 & 57.3 & 43.6 & 100 \\
\hline 9 & 63.5 & 65.9 & 41 & 53.3 & 68.9 & 33.3 \\
\hline 10 & 59.8 & 86.3 & 34 & 42.7 & 50.2 & 44.5 \\
\hline 11 & 47.6 & 50.4 & 44.7 & 58.4 & 62.6 & 37.1 \\
\hline 12 & 29.5 & 47.1 & 58 & 44.5 & 71.4 & 33.4 \\
\hline 13 & 42 & 49.8 & 41 & 47 & 60.5 & 40.9 \\
\hline 14 & 19.2 & 35.5 & 49.2 & 57.8 & 63.5 & 37 \\
\hline 15 & 21.2 & 31.6 & 49.2 & 52.1 & 72.6 & 31 \\
\hline 16 & 37.7 & 33.6 & 38.4 & 47 & 71.9 & 31.1 \\
\hline 17 & 28.1 & 36.2 & 41 & 41.6 & 73.9 & 32.4 \\
\hline 18 & 31.6 & 33.8 & 42.3 & 39.4 & 67.7 & 37.8 \\
\hline 19 & 29.5 & 35.5 & 35.5 & 50.2 & 55.6 & 46.1 \\
\hline 20 & 36.3 & 25.3 & 30.8 & 47.5 & 70 & 29.7 \\
\hline 21 & 0 & 39.9 & 37 & 52.1 & 59.3 & 33.5 \\
\hline 22 & 14.5 & 35.8 & 43.5 & 32.9 & 64 & 39.9 \\
\hline 23 & 34.4 & 0 & 51.3 & 41.6 & 76.6 & 25.8 \\
\hline 24 & 34.4 & 24.9 & 51.3 & 42 & 51.7 & 37.2 \\
\hline 25 & 15.4 & 19.2 & 57.1 & 38.9 & 62.1 & 25.9 \\
\hline 26 & 15.4 & 22.1 & 54.3 & 35.6 & 59.6 & 32.8 \\
\hline 27 & 19.9 & 17.2 & 32.4 & 38.2 & 80.1 & 30.3 \\
\hline 28 & 32.8 & 34.8 & 30.8 & 35 & 62.7 & 24.3 \\
\hline 29 & 28.1 & 31.9 & 32.4 & 39.5 & 57.3 & 22 \\
\hline 30 & 21.8 & 18.8 & 32.4 & 36.2 & 65.2 & 41.9 \\
\hline 31 & 29.9 & 36.2 & 30.8 & 33.1 & 55.1 & 29.1 \\
\hline 32 & 31.6 & 37.2 & 27.1 & 31.5 & 58.4 & 23.8 \\
\hline 33 & 29.5 & 16.3 & 39.7 & 32.5 & 64.8 & 24.1 \\
\hline 34 & 15.4 & 18.8 & 42.3 & 32.7 & 64.5 & 27.2 \\
\hline 35 & 18.5 & 32.6 & 37 & 26.4 & 58.4 & 29 \\
\hline 36 & 8.9 & 23.7 & 39.7 & 32.6 & 60.8 & 33.8 \\
\hline 37 & 17 & 59.8 & 27.1 & 41.8 & 19.3 & 40 \\
\hline 38 & 12.6 & 34.1 & 30.8 & 36.8 & 46.2 & 35.1 \\
\hline 39 & 33.6 & 27.4 & 20.5 & 29.7 & 61.9 & 25.3 \\
\hline 40 & 17 & 13.3 & 35.5 & 24.8 & 67.9 & 32.2 \\
\hline 41 & 20.5 & 24.9 & 32.4 & 31.3 & 52.1 & 26.8 \\
\hline 42 & 14.5 & 39.1 & 32.4 & 27.3 & 37.7 & 38.2 \\
\hline 43 & 18.5 & 34.5 & 30.8 & 37.6 & 34.9 & 27.7 \\
\hline 44 & 25.6 & 26.6 & 22.9 & 25.1 & 52.6 & 40.2 \\
\hline 45 & 16.2 & 16.3 & 29 & 37 & 56.3 & 26.6 \\
\hline 46 & 30.3 & 54.3 & 10.3 & 17.6 & 47.9 & 27.7 \\
\hline 47 & 19.9 & 25.3 & 22.9 & 30.6 & 51.8 & 34.9 \\
\hline 48 & 34.8 & 21.6 & 29 & 23.3 & 49.7 & 34.6 \\
\hline 49 & 0 & 31.7 & 35.5 & 23.4 & 53.9 & 26.2 \\
\hline 50 & 21.2 & 21 & 34 & 19.6 & 55.3 & 27.9 \\
\hline
\end{tabular}

TABLE III

ORIGINAL DATA FOR 50 UNIVERSITIES

Necessity: The proof is same to the proof of Property 1.

Similarly, we can prove the property of model $P_{2}$.

This completes the proof of Property 2.

Proof of Property 3: Assuming that $\exists h \in G, r^{*}\left(x_{h}\right) \notin$ $\left[\underline{r}_{w \in W}^{\mathrm{WA}}\left(x_{h}\right), \bar{r}_{w \in W}^{\mathrm{WA}}\left(x_{h}\right)\right]$, solutions of models $P_{1}$ and $P_{2}$ exist, which means that a manipulator can set strategic weight to achieve his/her desired ranking. However, according to the definition of ranking range under attribute weights $W$, $R_{w \in W}^{\mathrm{WA}}\left(x_{h}\right)=\left[\underline{r}_{w \in W}^{\mathrm{WA}}\left(x_{h}\right), \bar{r}_{w \in W}^{\mathrm{WA}}\left(x_{h}\right)\right]$ means the ranking of alternative manipulator $x_{h}$ vary in this range. The above two results are in contradiction.

Then, this completes the proof of Property 3.

\section{APPENDIX B}

See Table III. 


\section{REFERENCES}

[1] F. H. Barron and B. E. Barrett, "Decision quality using ranked attribute weights," Manag. Sci., vol. 42, no. 11, pp. 1515-1523, Nov. 1996.

[2] D. Ben-Arieh and T. Easton, "Multi-criteria group consensus under linear cost opinion elasticity," Decis. Support Syst., vol. 43, no. 3, pp. 713-721, Apr. 2007.

[3] D. Ben-Arieh, T. Easton, and B. Evans, "Minimum cost consensus with quadratic cost functions," IEEE Trans. Syst., Man, Cybern. A, Syst., Humans, vol. 39, no. 1, pp. 210-217, Jan. 2009.

[4] P. A. Bottomley and J. R. Doyle, "A comparison of three weight elicitation methods: Good, better, and best," Omega, vol. 29, no. 6, pp. 553-560, Dec. 2001.

[5] N. Capuano, F. Chiclana, H. Fujita, E. Herrera-Viedma, and V. Loia "Fuzzy group decision making with incomplete information guided by social influence," IEEE Trans. Fuzzy Syst., vol. 26, no. 3, pp. 1704-1718, Jun. 2018.

[6] M. Y. Cai, Y. Z. Lin, B. Han, C. J. Liu, and W. J. Zhang, "On a simple and efficient approach to probability distribution function aggregation," IEEE Trans. Syst., Man, Cybern., Syst., vol. 47, no. 9, pp. 2444-2453, Sep. 2017.

[7] A. Charnes, W. W. Cooper, and E. Rhodes, "Measuring the efficiency of decision making units," Eur. J. Oper. Res., vol. 2, no. 6, pp. 429-444, Nov. 1978.

[8] J. Castro, J. Lu, G. Q. Zhang, C. Y. Dong, and L. Martínez, "Opinion dynamics-based group recommender systems," IEEE Trans. Syst., Man, Cybern., Syst., to be published, doi: 10.1109/TSMC.2017.2695158.

[9] X. Chen, H. J. Zhang, and C. Y. Dong, "The fusion process with heterogeneous preference structures in group decision making: A survey," Inf. Fusion, vol. 24, pp. 72-83, Jul. 2015.

[10] W. D. Cook and M. Kress, "A data envelopment model for aggregating preference rankings," Manag. Sci., vol. 36, no. 11, pp. 1302-1310, 1990.

[11] M. Danielson, L. Ekenberg, and Y. He, "Augmenting ordinal methods of attribute weight approximation," Decis. Anal., vol. 11, no. 1, pp. 21-26, Mar. 2014.

[12] Y. C. Dong, Y. T. Liu, H. M. Liang, F. Chiclana, and E. Herrera-Viedma, "Strategic weight manipulation in multiple attribute decision making," Omega, vol. 75, no. 3, pp. 154-164, Mar. 2018.

[13] Y. C. Dong, H. J. Zhang, and E. Herrera-Viedma, "Integrating experts' weights generated dynamically into the consensus reaching process and its applications in managing non-cooperative behaviors," Decis. Support Syst., vol. 84, pp. 1-15, Apr. 2016.

[14] Y. C. Dong, H. J. Zhang, and E. Herrera-Viedma, "Consensus reaching model in the complex and dynamic MAGDM problem," Knowl. Based Syst., vol. 106, pp. 206-219, Aug. 2016.

[15] J. R. Doyle, R. H. Green, and P. A. Bottomley, "Judging relative importance: Direct rating and point allocation are not equivalent," Org. Behav. Human Decis. Process., vol. 70, no. 1, pp. 65-72, Apr. 1997.

[16] Z.-P. Fan, J. Ma, and Q. Zhang, "An approach to multiple attribute decision making based on fuzzy preference information on alternatives," Fuzzy Sets. Syst., vol. 131, no. 1, pp. 101-106, Oct. 2002.

[17] Z. W. Gong, X. X. Xu, F. L. Lu, L. S. Li, and C. Xu, "On consensus models with utility preferences and limited budget," Appl. Soft. Comput., vol. 35, pp. 840-849, Oct. 2015.

[18] Z. W. Gong, H. H. Zhang, J. Forrest, L. S. Li, and X. X. Xu, "Two consensus models based on the minimum cost and maximum return regarding either all individuals or one individual," Eur. J. Oper. Res., vol. 240, pp. 183-192, Jan. 2015.

[19] P. Gupta, C.-T. Lin, M. K. Mehlawat, and N. Grover, "A new method for intuitionistic fuzzy multiattribute decision making," IEEE Trans. Syst., Man, Cybern., Syst., vol. 46, no. 9, pp. 1167-1179, Sep. 2016.

[20] D. Horsky and M. R. Rao, "Estimation of attribute weights from preference comparisons," Manag. Sci., vol. 30, no. 7, pp. 801-822, Jul. 1984.

[21] S.-H. Kim and B.-S. Ahn, "Group decision making procedure considering preference strength under incomplete information," Comput. Oper. Res., vol. 24, no. 12, pp. 1101-1112, Dec. 1997.

[22] W. Q. Liu, Y. C. Dong, F. Chiclana, F. J. Cabrerizo, and E. Herrera-Viedma, "Group decision-making based on heterogeneous preference relations with self-confidence," Fuzzy Optim. Decis. Making, vol. 16, no. 4, pp. 429-447, Dec. 2017.

[23] Y. T. Liu, Y. C. Dong, F. Chiclana, F. J. Cabrerizo, and E. Herrera-Viedma, "Strategic weight manipulation in multiple attribute decision making in an incomplete information context," in Proc. IEEE Int. Conf. Fuzzy Syst. (FUZZ-IEEE), 2017, pp. 1-6.
[24] Y. Li, H. J. Zhang, and Y. C. Dong, "The interactive consensus reaching process with the minimum and uncertain cost in group decision making," Appl. Soft. Comput., vol. 60, pp. 202-212, Nov. 2017.

[25] A. Mateos, A. Jiménez, and S. Ríos-Insua, "Monte Carlo simulation techniques for group decision making with incomplete information," Eur. J. Oper. Res., vol. 174, no. 3, pp. 1842-1864, Nov. 2006.

[26] I. Palomares, L. Martínez, and F. Herrera, "A consensus model to detect and manage noncooperative behaviors in large-scale group decision making," IEEE Trans. Fuzzy Syst., vol. 22, no. 3, pp. 516-530, Jun. 2014.

[27] K. S. Park, K. S. Lee, Y. S. Eum, and K. Park, "Extended methods for identifying dominance and potential optimality in multi-criteria analysis with imprecise information," Eur. J. Oper. Res., vol. 134, no. 3, pp. 557-563, Nov. 2001.

[28] I. J. Pérez, F. J. Cabrerizo, S. Alonso, and E. Herrera-Viedma, "A new consensus model for group decision making problems with nonhomogeneous experts," IEEE Trans. Syst., Man, Cybern., Syst., vol. 44, no. 4, pp. 494-498, Apr. 2014.

[29] L. G. Pérez, F. Mata, F. Chiclana, G. Kou, and E. Herrera-Viedma, "Modelling influence in group decision making," Soft Comput., vol. 20, no. 4, pp. 1653-1665, Apr. 2016.

[30] D. Pekelman and S. K. Sen, "Mathematical programming models for the determination of attribute weights," Manag. Sci., vol. 20, no. 8 , pp. 1217-1229, 1974

[31] D. A. Pelta and R. R. Yager, "Decision strategies in mediated multiagent negotiations: An optimization approach," IEEE Trans. Syst., Man, Cybern. A, Syst., Humans, vol. 40, no. 3, pp. 635-640, May 2010.

[32] R. Roberts and P. Goodwin, "Weight approximations in multi-attribute decision models," J. Multi Crit. Decis. Anal., vol. 11, no. 6, pp. 291-303, Nov. 2002.

[33] T. L. Saaty, "The modern science of multicriteria decision making and its practical applications: The AHP/ANP approach," Oper. Res., vol. 61, no. 5, pp. 1101-1118, Sep. 2013.

[34] Academic Ranking of World Universities, Shanghai Jiao Tong Univ., Shanghai, China, 2017.

[35] L. E. Shirland, R. R. Jesse, R. L. Thompson, and C. L. Iacovou, "Determining attribute weights using mathematical programming," Omega, vol. 31, no. 6, pp. 423-437, Dec. 2003.

[36] K. Sugihara, H. Ishii, and H. Tanaka, "Interval priorities in AHP by interval regression analysis," Eur. J. Oper. Res., vol. 158, no. 3, pp. 745-754, Nov. 2004.

[37] Z. F. Tao, X. Liu, H. Y. Chen, and L. G. Zhou, "Using new version of extended t-norms and s-norms for aggregating interval linguistic labels," IEEE Trans. Syst., Man, Cybern., Syst., vol. 47, no. 12, pp. 3284-3298, Dec. 2017.

[38] Y.-M. Wang and K.-S. Chin, "The use of OWA operator weights for cross-efficiency aggregation," Omega, vol. 39, no. 5, pp. 493-503, 2011.

[39] J. Wu, F. Chiclana, and E. Herrera-Viedma, "Trust based consensus model for social network in an incomplete linguistic information context," Appl. Soft. Comput., vol. 35, pp. 827-839, Oct. 2015.

[40] J. Wu and F. Chiclana, "A social network analysis trust-consensus based approach to group decision-making problems with interval-valued fuzzy reciprocal preference relations," Knowl. Based Syst., vol. 59, pp. 97-107, Mar. 2014.

[41] X.-H. Xu, Z.-J. Du, and X.-H. Chen, "Consensus model for multi-criteria large-group emergency decision making considering non-cooperative behaviors and minority opinions," Decis. Support Syst., vol. 79, pp. 150-160, Nov. 2015.

[42] R. R. Yager, "Defending against strategic manipulation in uninormbased multi-agent decision making," Eur. J. Oper. Res., vol. 141, no. 1, pp. 217-232, Aug. 2002.

[43] R. R. Yager, "Penalizing strategic preference manipulation in multi-agent decision making," IEEE Trans. Fuzzy Syst., vol. 9, no. 3, pp. 393-403, Jun. 2001.

[44] R. R. Yager, "On ordered weighted averaging aggregation operators in multi-criteria decision making," IEEE Trans. Syst., Man, Cybern., Syst., vol. 18, no. 1, pp. 183-190, Jan./Feb. 1988.

[45] R. R. Yager, "Multi-criteria decision making with interval criteria satisfactions using the golden rule representative value," IEEE Trans. Fuzzy Syst., vol. 26, no. 2, pp. 1023-1031, Apr. 2018.

[46] R. R. Yager and N. Alajlan, "Multicriteria decision-making with imprecise importance weights," IEEE Trans. Fuzzy Syst., vol. 22, no. 4, pp. 882-891, Aug. 2014.

[47] C. L. Hwang and K. Yoon, Multiple Attribute Decision Making: Methods and Applications. Berlin, Germany: Springer, 1981. 
[48] S. H. Zanakis, A. Solomon, N. Wishart, and S. Dublish, "Multi-attribute decision making: A simulation comparison of select methods," Eur. J. Oper. Res., vol. 107, no. 3, pp. 507-529, Jun. 1998.

[49] H. J. Zhang, Y. C. Dong, and X. Chen, "The 2-rank consensus reaching model in the multigranular linguistic multiple-attribute group decisionmaking," IEEE Trans. Syst., Man, Cybern., Syst., to be published, doi: $10.1109 /$ tsmc.2017.2694429.

[50] H. J. Zhang, Y. C. Dong, and E. Herrera-Viedma, "Consensus building for the heterogeneous large-scale GDM with the individual concerns and satisfactions," IEEE Trans. Fuzzy Syst., vol. 26, no. 2, pp. 884-898, Apr. 2018.

[51] G. Q. Zhang, Y. C. Dong, Y. F. Xu, and Y. H. Li, "Minimum-cost consensus models under aggregation operators," IEEE Trans. Syst., Man, Cybern. A, Syst., Humans, vol. 41, no. 6, pp. 1253-1261, Nov. 2011.

[52] Z. Zhang, C. H. Guo, and L. Martínez, "Managing multigranular linguistic distribution assessments in large-scale multiattribute group decision making," IEEE Trans. Syst., Man, Cybern., Syst., vol. 47, no. 11, pp. 3063-3076, Nov. 2017.

[53] B. W. Zhang, Y. C. Dong, and Y. F. Xu, "Maximum expert consensus models with linear cost function and aggregation operators," Comput. Ind. Eng., vol. 66, no. 1, pp. 147-157, 2013.

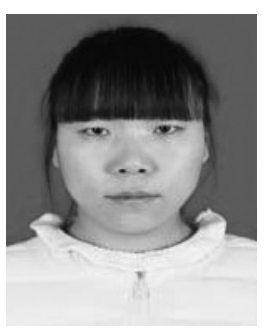

Yating Liu received the M.S. degree in information and computing science from the Beifang University of Nationalities, Yinchuan, China, in 2013. She is currently pursuing the Ph.D. degree in management with the Business School, Sichuan University, Chengdu, China.

Her current research interests include multiple attribute decision making and decision support systems.

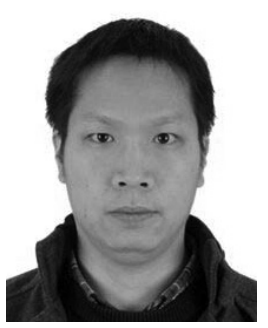

Yucheng Dong received the B.S. and M.S degrees in mathematics from Chongqing University, Chongqing, China, in 2002 and 2004, respectively, and the Ph.D. degree in management from Xi'an Jiaotong University, Xi'an, China, in 2008.

$\mathrm{He}$ is currently a Professor with the Business School, Sichuan University, Chengdu, China. He has published over 90 international journal papers in Decision Support Systems, the European Journal of Operational Research, the IEEE TRANSACTIONS ON Big Data, the IEEE TRANSACTIONS ON Cybernetics, the IEEE Transactions on FuZzy Systems, the IEEE Transactions on Systems, Man, And Cybernetics: Systems, and Omega. His current research interests include group decision making, linguistic decision making, opinion dynamics, and social network decision making.

Dr. Dong is an Editorial Board Member of Information Fusion and an Area Editor of Computers \& Industrial Engineering.

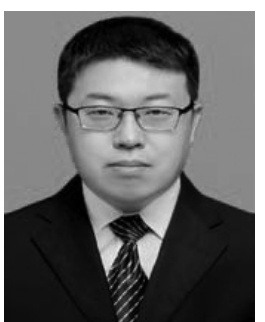

Haiming Liang received the Ph.D. degree in management from Northeastern University, Shenyang, China, in 2014

$\mathrm{He}$ is an Associate Research Fellow with the Business School, Sichuan University, Chengdu, China. He has published several international journal papers in the European Journal of Operational Research, Omega, Computers \& Industrial Engineering, Information Fusion, the Journal of Artificial Societies and Social Simulation, and the Journal of the Operational Research Society. His current research interests include multiple attribute decision making and opinion dynamics.

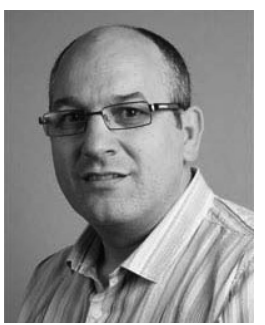

Francisco Chiclana received the B.Sc. and $\mathrm{Ph} . \mathrm{D}$. degrees in mathematics from the University of Granada, Granada, Spain, in 1989 and 2000, respectively.

He is currently a Professor of Computational Intelligence and Decision Making with the School of Computer Science and Informatics, Faculty of Technology, De Montfort University, Leicester, U.K., and an Honorary Professor with the Department of Mathematics, University of Leicester.

Dr. Chiclana is an Associate Editor for several journals, such as the IEEE TRANSACTIONS ON Systems, MAN, AND CybERnETICS: Systems, Information Sciences, and Knowledge-Based Systems. He has organized and chaired special sessions/workshops in many major international conferences in research areas relevant to fuzzy preference modeling, decision-making, decision support systems, consensus, recommender systems, and social networks.

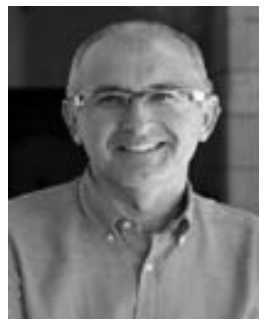

Enrique Herrera-Viedma received the M.Sc. and Ph.D. degrees in computer science from the University of Granada, Granada, Spain, in 1993 and 1996, respectively.

$\mathrm{He}$ is a Professor of Computer Science and the Vice-President for Research and Knowledge Transfer with the University of Granada. He has over 13000 Web of Science citations with an $H$-index of 62 and over 24000 Google Scholar citations with an $H$-index of 75 . He has been identified as one of the World's Most Influential Researchers by the Shanghai Center and Thomson Reuters/Clarivate Analytics in both Computer Science and Engineering in 2014, 2015, 2016, and 2017. His current research interests include group decision making, consensus models, linguistic modeling, aggregation of information, information retrieval, bibliometrics, digital libraries, Web quality evaluation, recommender systems, and social media.

Dr. Herrera-Viedma is Vice-President for Publications in the SMC Society and an Associate Editor for several journals, such as the IEEE TRANSACTIONS ON FUZZY SYSTEMS, the IEEE TRANSACTIONS ON SYSTEMS, MAN, AND CYBERNETICS: SYSTEMS, the IEEE TRANSACTIONS ON INTELLIGENT TRANSPORTATION SYSTEMS, Information Sciences, Applied Soft Computing, Soft Computing, Fuzzy Optimization and Decision Making, and Knowledge-Based Systems. 